\title{
Oxygen Isotopic Composition of coarse- and fine-grained material from Comet 81P/Wild 2
}

\author{
Ryan C. Ogliore ${ }^{\mathrm{a}}$, Kazuhide Nagashima ${ }^{\mathrm{a}}$, Gary R. Huss ${ }^{\mathrm{a}}$, Andrew J. Westphal ${ }^{\mathrm{b}}$, Zack \\ Gainsforth $^{\mathrm{b}}$, Anna L. Butterworth ${ }^{\mathrm{b}}$ \\ ${ }^{a}$ Hawai'i Institute of Geophysics and Planetology, University of Hawai'i at Mānoa, Honolulu, HI 96822, \\ USA \\ ${ }^{b}$ Space Sciences Laboratory, University of California at Berkeley, Berkeley, CA 94720, USA
}

\begin{abstract}
Individual particles from comet 81P/Wild 2 collected by NASA's Stardust mission vary in size from small sub- $\mu \mathrm{m}$ fragments found in the walls of the aerogel tracks, to large fragments up to tens of $\mu \mathrm{m}$ in size found towards the termini of tracks. The comet, in an orbit beyond Neptune since its formation, retains an intact a record of early-Solar-System processes that was compromised in asteroidal samples by heating and aqueous alteration. We measured the $\mathrm{O}$ isotopic composition of seven Stardust fragments larger than $\sim 2 \mu \mathrm{m}$ extracted from five different Stardust aerogel tracks, and 63 particles smaller than $\sim 2 \mu \mathrm{m}$ from the wall of a Stardust track. The larger particles show a relatively narrow range of $\mathrm{O}$ isotopic compositions that is consistent with ${ }^{16} \mathrm{O}$-poor phases commonly seen in meteorites. Many of the larger Stardust fragments studied so far have chondrule-like mineralogy which is consistent with formation in the inner Solar System. The fine-grained material shows a very broad range of O isotopic compositions $\left(-70 \%<<\Delta^{17} \mathrm{O}<+60 \%\right)$ suggesting that Wild 2 fines are either primitive outer-nebula dust or a very diverse sampling of inner Solar System compositional reservoirs that accreted along with a large number of inner-Solar-System rocks to form comet Wild 2.

Keywords: comets, solar nebula, Jupiter-family comets, Wild 2, oxygen isotopes, cosmochemistry
\end{abstract}

\section{Introduction}

Oxygen isotopic compositions are traditionally reported in delta notation - the partsper-thousand deviations of the stable $\mathrm{O}$ isotope ratios from standard mean ocean water $\left(\left({ }^{18} \mathrm{O} /{ }^{16} \mathrm{O}\right)_{\mathrm{SMOW}}=0.0020052\right.$ and $\left.\left({ }^{17} \mathrm{O} /{ }^{16} \mathrm{O}\right)_{\mathrm{SMOW}}=0.00038288\right):$ 


$$
\begin{aligned}
& \delta^{18} \mathrm{O}=\left(\frac{{ }^{18} \mathrm{O} /{ }^{16} \mathrm{O}}{\left({ }^{18} \mathrm{O} /{ }^{16} \mathrm{O}\right)_{\text {SMOW }}}-1\right) \times 1000 \\
& \delta^{17} \mathrm{O}=\left(\frac{{ }^{17} \mathrm{O} /{ }^{16} \mathrm{O}}{\left({ }^{17} \mathrm{O} /{ }^{16} \mathrm{O}\right)_{\text {SMOW }}}-1\right) \times 1000
\end{aligned}
$$

Most terrestrial processes that fractionate one isotope of an element relative to another are due to the fact that vibrational frequencies, bond strengths, and diffusion coefficients depend on an isotope's mass. Such mass-dependent fractionation, arising from equilibrium or kinetic effects, causes ${ }^{18} \mathrm{O} /{ }^{16} \mathrm{O}$ to change approximately twice as much as ${ }^{17} \mathrm{O} /{ }^{16} \mathrm{O}$. On an oxygen three-isotope plot of $\delta^{17} \mathrm{O}$ vs. $\delta^{18} \mathrm{O}$, most terrestrial samples fall along a line of slope $\sim 0.52$, called the terrestrial fractionation line (TFL). However, some extraterrestrial samples do not follow this trend. Calcium-aluminum inclusions, chondrules, and amoeboid olivine aggregates found in primitive chondritic meteorites fall on a slope $\sim 1$ line called the carbonaceous chondrite anhydrous mineral (CCAM) line (Clayton et al., 1977). The difference between the measured $\delta^{17} \mathrm{O}$ and the TFL is defined as:

$$
\Delta^{17} \mathrm{O}=\delta^{17} \mathrm{O}-0.52 \times \delta^{18} \mathrm{O}
$$

Refractory components like calcium-aluminum inclusions typically have a lower (i.e. more negative) $\Delta{ }^{17} \mathrm{O}$ than chondrules (low $\Delta^{17} \mathrm{O}$ objects are also called " ${ }^{16} \mathrm{O}$-rich"). However, most rocky inner Solar System materials (Earth, Moon, Mars, and bulk asteroids) are close to $\delta^{17,18} \mathrm{O}=(0,0)$. The $\mathrm{O}$ composition of the Sun, inferred from measurements of returned samples of the solar wind, is very ${ }^{16} \mathrm{O}$-rich: $\delta^{17,18} \mathrm{O}=(59.1 \%, 58.5 \%)$ (McKeegan et al., 2011). Photochemical processing (e.g. Clayton, 2002) has been suggested to explain the CCAM line by mass-independent isotope fractionation (processes that enrich ${ }^{18} \mathrm{O} /{ }^{16} \mathrm{O}$ and ${ }^{17} \mathrm{O} /{ }^{16} \mathrm{O}$ equally), but the large difference between the ${ }^{16} \mathrm{O}$-rich Sun and the ${ }^{16} \mathrm{O}$-poor inner Solar System solids would require such a process to be extremely efficient. It is more plausible that different reservoirs of O isotopic compositions were inherited from the Sun's parent molecular cloud (Huss, 2012), and the CCAM line reflects mixing between reservoirs. Oxygen isotopic 
measurements of primitive Solar System materials could yield insight into the composition of the dust and gas making up the protosolar molecular cloud.

The Moon, terrestrial planets, and large asteroids underwent melting, and differentiation due to accretional and radiogenic heating, so the O isotopic signature of the Solar System's building blocks are lost in these objects. Some "primitive" meteorites from smaller asteroids avoided significant aqueous and thermal processing. Analyses of these samples give us insight into, for example, the high-temperature refractory objects that were the first to form in the solar nebula, and the inner Solar System abundance of isotopically anomalous dust which formed around other stars (presolar grains).

However, outer Solar System bodies may retain pristine dust from the solar nebula along with ices and other volatile species that were vaporized closer to the Sun. Jupiter-family comets, in cold storage nearly all of their lives, preserved the $\mathrm{O}$ isotopic composition of the materials that originally accreted to form the comet in the outer Solar System. The diversity of $\mathrm{O}$ isotopic reservoirs inherited from the protosolar molecular cloud may still be found in cometary particles. Additionally, cometary material may contain a higher abundance, or additional types, of presolar grains than what is seen in primitive meteorites. More fragile presolar grains can be easily destroyed in the solar nebular or in an asteroid; the abundance and types of presolar grains in comets may reflect the true contribution of this exotic material to the solar nebula.

NASA's Stardust mission returned samples from the Jupiter-family comet 81P/Wild 2 in 2006 for laboratory study (Brownlee et al., 2006). Impacting cometary particles were collected at $\sim 6 \mathrm{~km} / \mathrm{s}$ in aerogel, which acted somewhat as a size-sorting sieve, with larger and more robust rock fragments, called terminal particles, found at the ends of tracks, and fine-grained material closer to the space-exposed aerogel surface in the track bulb. The terminal particles, protected by their thermal inertia, were collected mostly intact whereas the fines were intimately mixed with aerogel (e.g. Stodolna et al., 2012).

The terminal particles are the easiest objects to study due to their larger size, better preservation, and easier sample preparation. Many of the terminal particles studied so far 
have a high-temperature origin, likely in the inner Solar System: they are large $(>5 \mu \mathrm{m})$ single crystals (e.g. Track C2115,22,20: terminal particle, Joswiak et al., 2012), or have mineralogy consistent with fragments of chondrules (e.g. Track C2054,0,35,6: "Torajiro", Nakamura et al., 2008), calcium-aluminum inclusions (e.g. Track C2054,4,25: "Inti", Simon et al., 2008), metal (e.g. Track C2044,41: "Simieo", Westphal et al., 2012), and other phases.

Though a relatively small number of analyses have been performed on comet Wild 2 samples, it appears that the large terminal particles show more compositional diversity than similar objects in a given type of meteorite. Measurements of the $\mathrm{FeO}$ and $\mathrm{MnO}$ composition of Wild 2 ferrous olivine do not show a clear linear relationship as do ferrous olivines from specific classes of meteorites (Brownlee, 2014), indicating that Wild 2 olivines could not have originated from a small number of chondrite parent bodies. While the presolar grain abundance of comet Wild 2 has been difficult to precisely determine due to the small sample size and capture effects, the large majority of Wild 2 particles do not show extreme isotopic anomalies. The oxygen isotopic composition of $>3 \mu \mathrm{m}$ ferromagnesian crystalline silicates in Wild 2 show a $\Delta^{17} \mathrm{O}$ dependence on $\mathrm{Mg}$ content that is similar to $\mathrm{CR}$ chondrite chondrules (Nakashima et al., 2012a), though Wild 2 has a much larger relative abundance of FeO-rich particles than CR chondrites (Zolensky et al., 2006).

Asteroids accreted local material of relatively homogenous composition, as demonstrated by the diagnostic properties of meteorites (e.g. chondrule size, matrix fraction, oxygen isotopic composition) that allows different meteorites to be grouped together as originating from the same parent asteroid. Conversely, the larger particles in comet Wild 2 appear to have formed elsewhere (Brownlee et al., 2012), that is, material was transported from the inner Solar System (e.g. Ciesla, 2007) to beyond the orbit of Neptune where Jupiter-family comets like Wild 2 probably formed.

The chemical compositions of the fine-grained material located in the bulbous cavity immediately after the point of impact of a Stardust track have been analyzed by Stodolna et al. (2012). Despite difficulties arising from sample alteration due to high-speed capture into aerogel, the fine-grained bulb material appears to be approximately solar in its average 
Fe-Mg-S composition, but heterogeneous on a particle-by-particle basis (Westphal et al., 2009). These are the expected characteristics of primitive dust that has not been significantly thermally altered in the solar nebula or in a parent body.

Fine-grained cometary dust captured in the track bulb could be the missing "pristine" nebular material that was expected to make up the bulk of comet Wild 2 before the Stardust mission returned with samples. The fines are either (1) outer-nebula dust, locally present in the outer Solar System where comet Wild 2 formed and likely representative of unprocessed particles that made up our parent molecular cloud, or (2) inner Solar System material, possibly related to meteorite matrix, that was transported to the outer disk along with the terminal particles, or a combination of (1) and (2). Fines from source 1) would be rich in circumstellar grains with nucleosynthetic isotopic anomalies and other material inherited from the Solar System's parent molecular cloud, whereas those from source (2) would be similar in composition to the fine-grained matrix of primitive meteorites (material that did not experience thermal or aqueous processing on its asteroidal parent body).

To gain insight into the origins of coarse-grained $(>2 \mu \mathrm{m})$ and fine-grained $(<2 \mu \mathrm{m})$ material from comet Wild 2, we measured the oxygen isotopic composition of terminal particles and fine-grained bulb material of cometary matter returned by NASA's Stardust mission.

\section{Description of Wild 2 Particles}

\subsection{Individual fragments}

We characterized the mineralogy and measured the O isotopic composition of seven Stardust fragments larger than $\sim 2 \mu \mathrm{m}$ extracted from five different Stardust aerogel tracks. The seven fragments are described individually below and the tracks from which they were extracted are described in Table 1. The pyroxene and olivine phases we analyzed all had relatively FeO-rich compositions, i.e. all had mol. \% fayalite $>10$. This lack of MgO-rich silicates is not surprising, since it has been shown (e.g., Frank et al., 2014) that Wild 2 has a flatter distribution of fayalite content compared to matrix grains in chondrites, and lacks the $\mathrm{MgO}$-rich silicates (mol. \% fayalite $<5$ ) that are abundant in many meteorite classes. 


\begin{tabular}{lccc}
\hline Track & Type $^{\dagger}$ & Length $(\mathrm{mm})$ & Particles Analyzed \\
\hline & & & \\
C2052,74 & B & 8.0 & Iris, Callie, Tintin, bulb material \\
C2061,113 & B & 1.5 & Pooka \\
C2035,105 & B & 2.2 & Caligula \\
C2009,77 & B & 2.5 & The Butterfly \\
C2062,162 & A & 2.9 & Cecil \\
\hline
\end{tabular}

Table 1: Track Information. ${ }^{\dagger}$ Stardust cometary track types are described by Burchell et al. (2008).

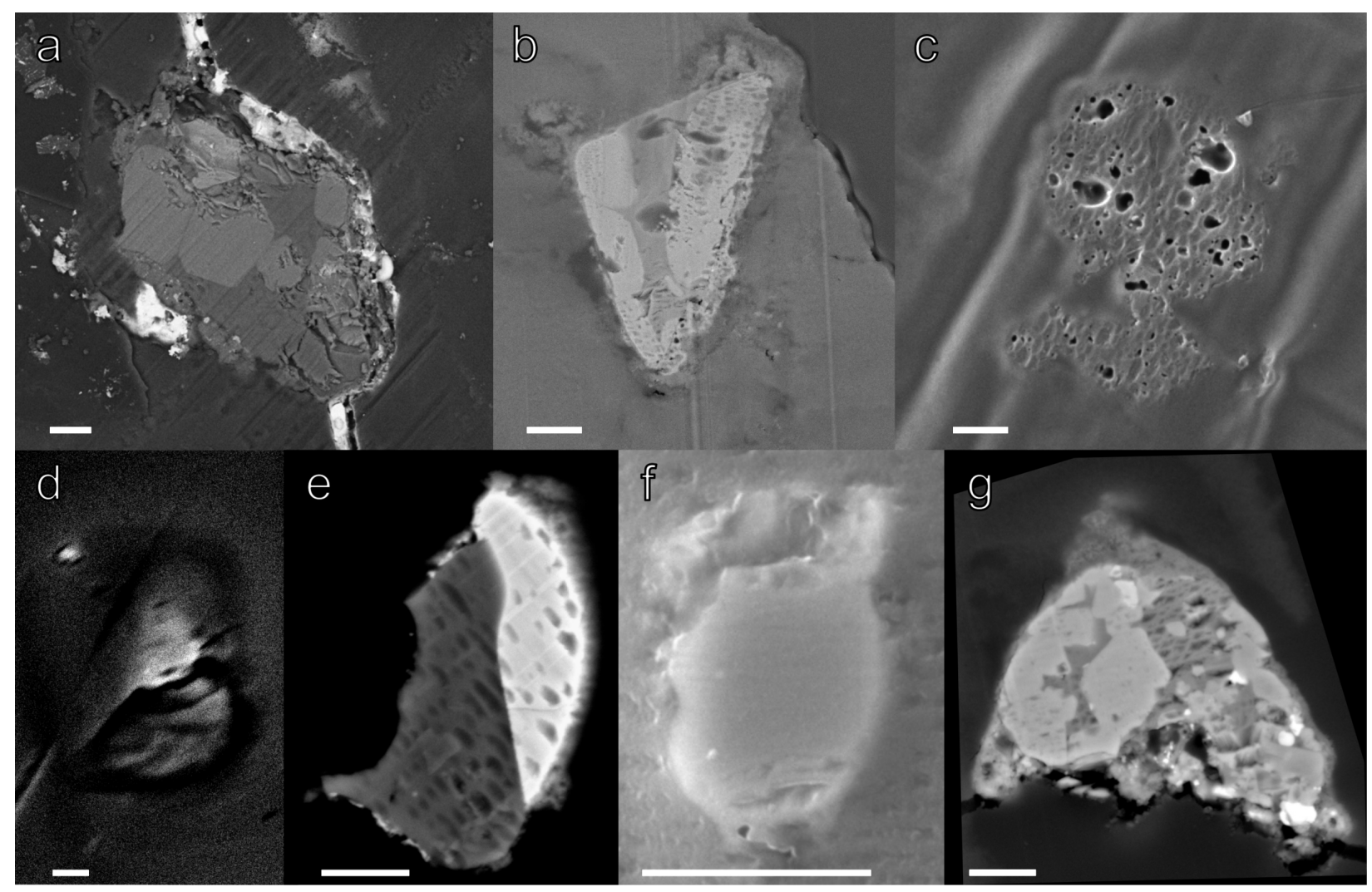

Figure 1: Electron microscope images ( $\mathrm{SE}=$ secondary electrons, $\mathrm{BSE}=$ backscattered electrons) of seven Stardust fragments measured individually. White scale bar is $2 \mu \mathrm{m}$ in all panels. a) Iris (BSE), b) Callie (BSE), c) Tintin (SE), d) Pooka (BSE), e) Caligula (BSE), f) The Butterfly (SE), g) Cecil (BSE).

Iris: Iris is a type-II chondrule fragment from Track C2052,74, $23 \times 10 \mu \mathrm{m}$ in size, described in detail in Ogliore et al. (2012). This particle contains Fe-rich olivines, chromite, and plagioclase in a $\mathrm{SiO}_{2}$-rich, mostly glassy mesostasis.

Callie: Callie is a type-II chondrule fragment from Track C2052,74, $10 \times 6 \mu \mathrm{m}$ in size, 


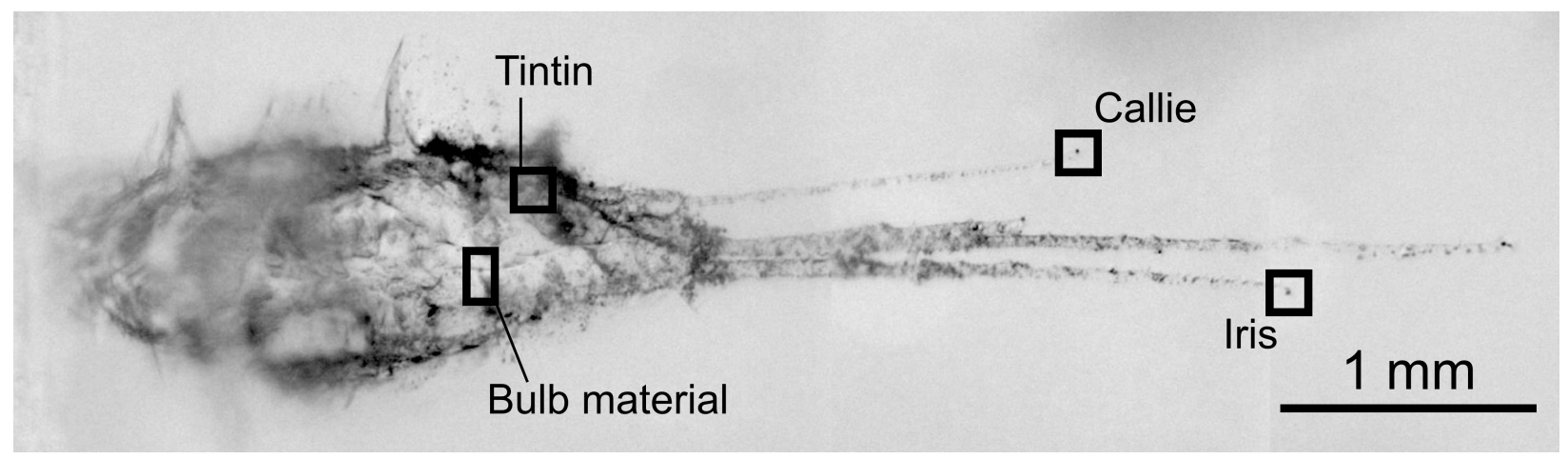

Figure 2: Photomicrograph of Stardust track C2052,74. Positions of Iris, Callie, Tintin (approximate), and the bulb material (approximate) are indicated.

possibly related to Iris, described in detail in Stodolna et al. (2013). Like Iris, Callie contains subgrains of Fe-rich olivine, plagioclase, and chromite in a subcrystalline mesostasis.

Tintin: Tintin is a $10 \times 8 \mu \mathrm{m}$ amorphous Fe-rich, Al-bearing silicate particle with a vesiculated texture from the bulb of track C2052,74.

Pooka: Pooka is a $3 \times 3 \mu \mathrm{m}$ particle from track $\mathrm{C} 2061,113$ and consists mostly of pyroxene $\left(\mathrm{En}_{80}\right)$.

Caligula: Caligula is the largest terminal particle in track C2035,105. It is a $12 \times 6 \mu \mathrm{m}$ assemblage of pyrrhotite and amorphous silicate, with inclusions of pyroxene and sulfides (Gainsforth et al., 2013).

The Butterfly: The Butterfly is a $3 \times 2 \mu \mathrm{m}$ particle from the bulb region of track C2009,77. It consists mostly of pyroxene $\left(\mathrm{En}_{88}\right)$, with sub- $\mu \mathrm{m}$ Al- and Na-rich phases.

Cecil: Cecil is a terminal particle from track C2062,162. It is $10 \times 8 \mu \mathrm{m}$ in size and is composed of Fe-rich olivine $\left(\mathrm{Fo}_{66}, \mathrm{Fo}_{68}\right)$, Ni-rich and Ni-poor sulfides, spinel, and pyroxene, with an amorphous silicate groundmass (Gainsforth et al., 2014).

\subsection{Bulb of Track 74}

We measured the $\mathrm{O}$ isotopic compositions of 63 particles from the bulb region of Stardust track C2052,74 (Figure 2). This is a Type B track (initial bulbous cavity tapering into one or more long, narrow tracks, see Burchell et al., 2008) with a total length of $\sim 6 \mathrm{~mm}$. The widest part of the bulb is $2.5 \times 1 \mathrm{~mm}$. The fine-grained material in the bulb was identified by its Mg-rich composition compared to the surrounding aerogel in an Energy Dispersive X-ray 
Spectroscopy (EDS) map. The size distribution of the 63 measured fragments is shown in Figure 3. The $\mathrm{Mg}, \mathrm{Al}$, and $\mathrm{Fe}$ content of the fragments relative to $\mathrm{O}$ was measured by ion probe.

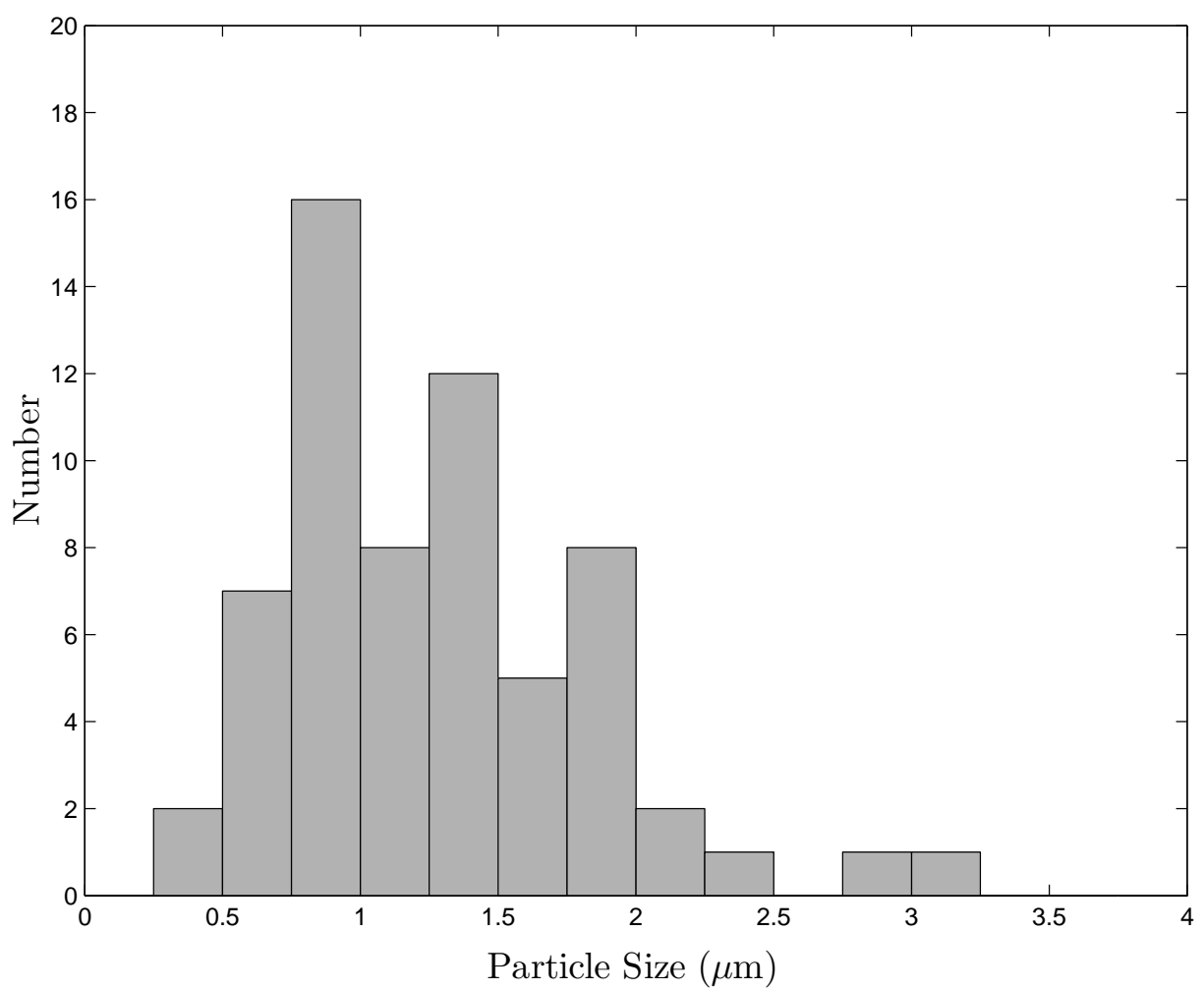

Figure 3: The size distribution (equivalent diameter) of the 63 measured Stardust fragments embedded in aerogel, extracted from the bulb of track C2052,74. Sizes were calculated from $\mathrm{Mg}-$-, Al-, or Fe-rich regions in the five scanning ion images shown in Figure 6.

\section{Sample Preparation}

The individual fragments (Section 2.1) and Track 74 bulb particles (Section 2.2) were prepared differently, as described below.

\subsection{Individual Fragments: Sample Preparation}

Stardust tracks were extracted from their aerogel tiles as keystones (Westphal et al., 2004) using glass needles robotically controlled by micromanipulators. After synchrotron analyses of fragments in the track (X-ray fluorescence mapping and Fe K-edge X-ray absorption 
near-edge spectroscopy, see Westphal et al., 2009), the keystone is then dissected further to remove the fragment of interest in a thin $(\sim 100 \mu \mathrm{m})$ wafer of aerogel, which was embedded in epoxy and attached to the end of an epoxy bullet. Approximately 25 (volume) $\%$ of the particle was ultramicrotomed into slices $\sim 100 \mathrm{~nm}$ thick and placed on transmission electron microscopy (TEM) grids with $10 \mathrm{~nm}$ amorphous carbon substrates, for analysis by TEM and synchrotron-based scanning transmission X-ray microscopy (STXM). The remaining 75\% of the particle remained in the epoxy bullet, its cross section exposed (a "potted butt") for SIMS (secondary ion mass spectrometry) isotope measurements.

The cylindrical potted butt was mounted in a holder specifically developed for SIMS analyses. Our goal with this holder was to mount the potted butt without modification and make it possible to measure the isotopic compositions of the minerals exposed in the potted butt while retaining the ability to make additional microtome sections and to expose fresh surfaces for isotopic measurements (Westphal et al., 2011). The mount consists of two components (Figure 4): a Cameca ims 1280-compatible 1-inch aluminum round (2.54-cm diameter by 1-cm thick), which held the potted butt at a fixed altitude, and a "buckler", which simultaneously surrounds the particle with a flat conductive plane, matching the altitude of the particle, and integrates a ring of five unique mineral standards populating 12 positions around the sample. The multiple use of the five standards in the buckler allowed for the determination of any instrumental fractionation effects that depend on position in the buckler. No such instrumental fractionation effects were found. The buckler consists of a thin $\mathrm{Al}$ disk with embedded standards, polished to a mirror finish, with a central $3 \mathrm{~mm}$ hole. We mounted a Au-coated, 500 nm-thick $\mathrm{Si}_{3} \mathrm{~N}_{4}$ membrane in which we had previously drilled a $300 \mu \mathrm{m}$ hole using an ion mill, over the hole in the $\mathrm{Al}$ disk. A motorized, encoded micromanipulator with $200 \mathrm{~nm}$ resolution (Sutter MP-285) was used to integrate the complete assembly, since $\sim 1 \mu \mathrm{m}$ precision is required in the three-dimensional alignment of the $\mathrm{Si}_{3} \mathrm{~N}_{4}$ window with the particle. After assembly the sample was flat due to the ultramicrotomed face of the epoxy plateau except for a micron-sized step at the edge of the hole in the $\mathrm{Si}_{3} \mathrm{~N}_{4}$ membrane more than $150 \mu \mathrm{m}$ away from the Stardust particle. 


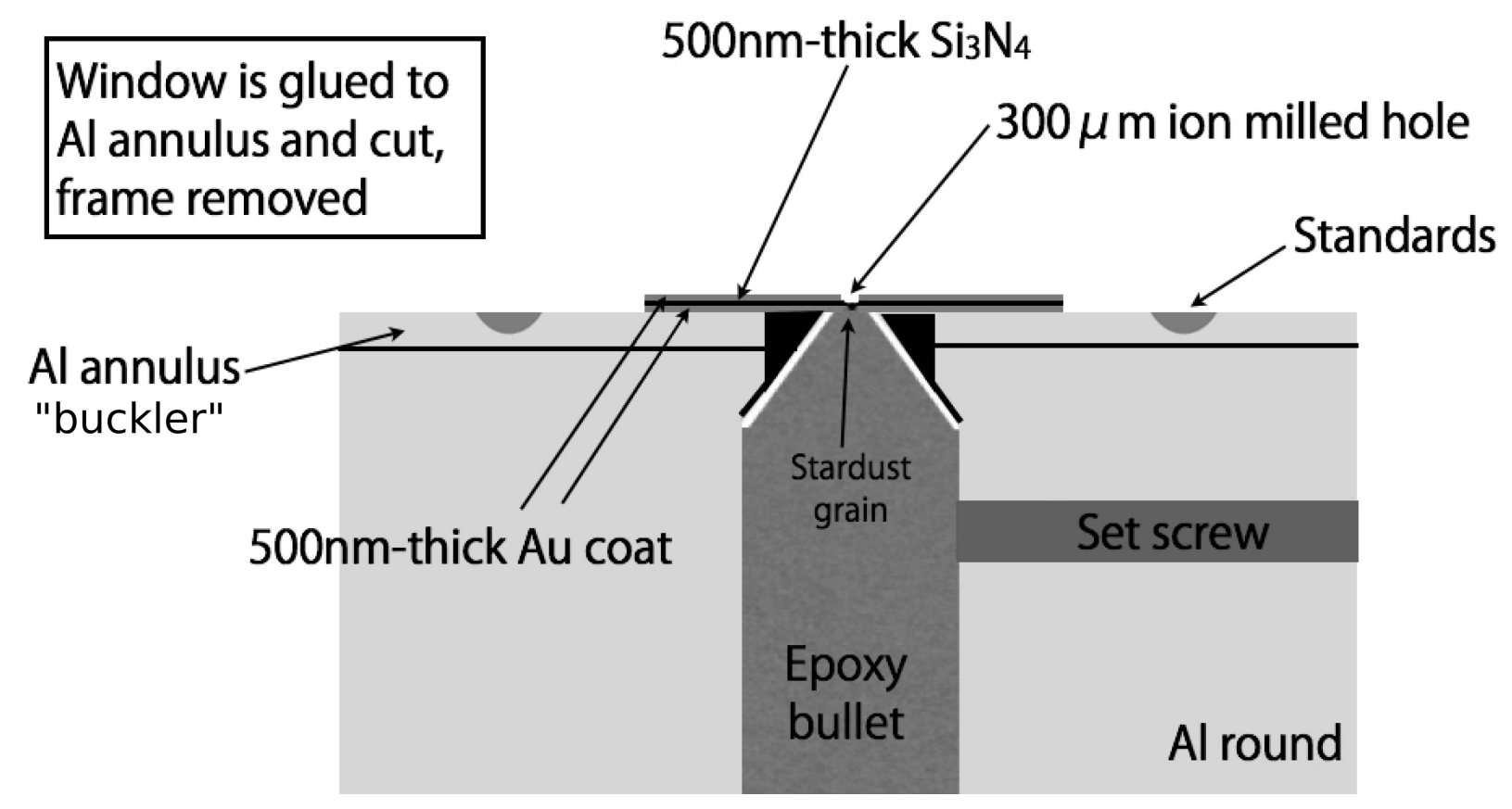

Figure 4: Reversible sample holder developed to make isotopic measurements of potted butts of Stardust particles by ion probe without remounting.

\subsection{Bulb of Track 74: Sample Preparation}

We extracted a $1 \mathrm{~mm}^{2}$ section from the wall of Stardust track C2052,74 using glass needles robotically controlled by micromanipulators. With a Teflon-coated anvil and an Instron press with a plunger calibrated for force, we compressed the aerogel sample into indium, which is pliable and electrically conductive. The aerogel porosity was sufficiently reduced to allow a conductive $\sim 50 \mathrm{~nm} \mathrm{Au}$ coat to be applied. The samples were then mounted under an $\mathrm{Au}$ coated $\mathrm{Si}_{3} \mathrm{~N}_{4}$ window with a $\sim 400 \mu \mathrm{m}$ ion-milled hole (Figure $5 \mathrm{a}$ ), similar to that described in Section 3.1, to create a flat, conducting surface for SIMS analysis (Westphal et al., 2011). We mapped the compressed aerogel in the University of Hawai'i's JEOL JXA-8500 fieldemission gun electron probe microanalyzer to identify regions rich in $\mathrm{Mg}$, which indicates the presence of cometary material (Figure $5 \mathrm{~b}$ ). We identified five regions to analyze for $\mathrm{O}$ isotopes by SIMS. Additionally, we prepared two standards to assess the accuracy of our SIMS O isotope measurements: San Carlos olivine (shot into aerogel at $\sim 6 \mathrm{~km} / \mathrm{s}$, extracted as a keystone, pressed into indium) and spinel separates from the Allende meteorite (pressed into aerogel, then pressed into indium). The San Carlos olivine has O isotopic composition: 
$\delta^{18} \mathrm{O}=5.25, \delta^{17} \mathrm{O}=2.73$; the Allende spinels have compositions that are well off the TFL: $\delta^{17,18} \mathrm{O} \approx-40 \%$ (Makide et al., 2009a).
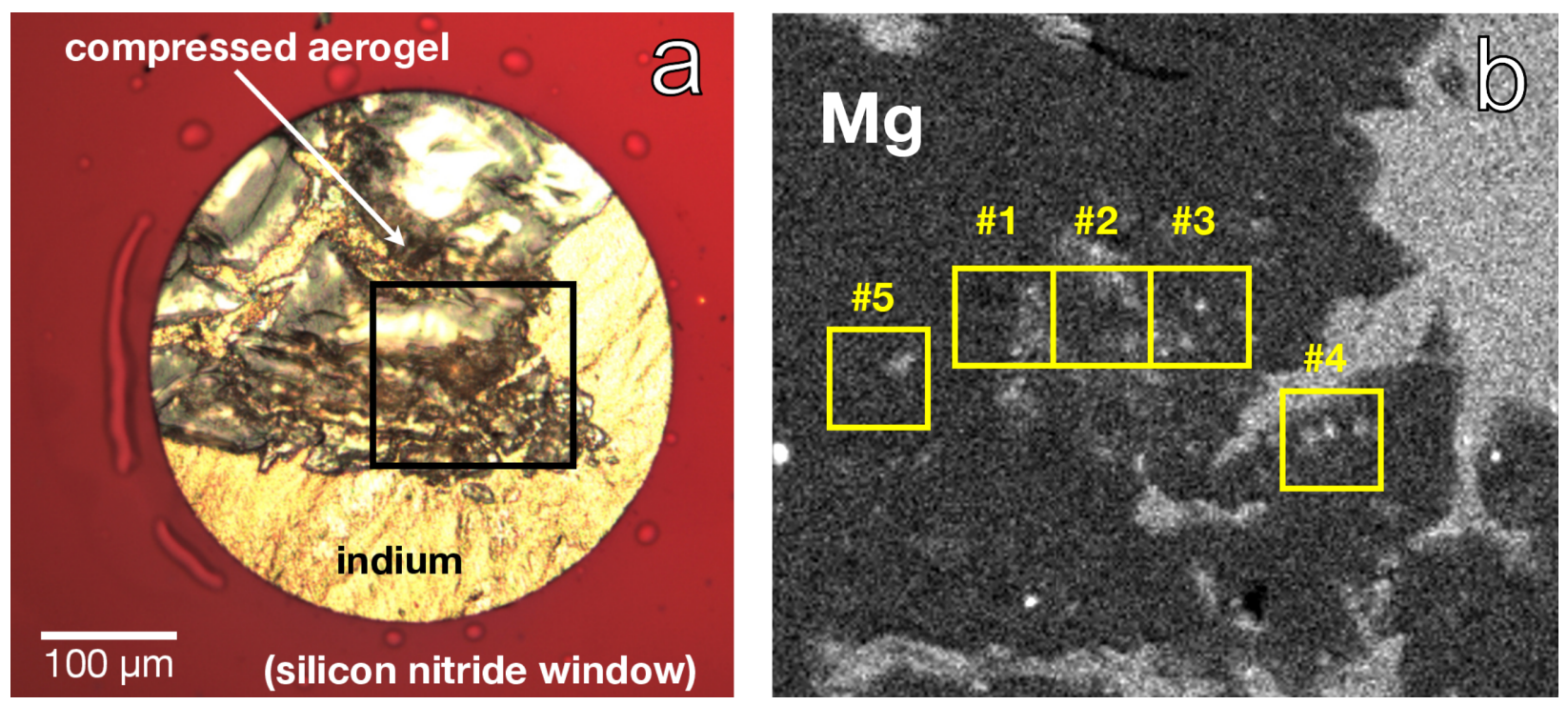

Figure 5: a) Compressed aerogel mounted for ion probe analysis. Area of Mg elemental map shown in b) is indicated by black rectangle. b) Mg elemental map of the region of compressed aerogel. The five $15 \times 15 \mu \mathrm{m}$ analysis areas are outlined. False detection of $\mathrm{Mg}$ in indium surrounding the aerogel is due to the increased bremsstrahlung there.

\section{Analytical Techniques}

The individual fragments (Section 2.1) and Track 74 bulb particles (Section 2.2) were measured using different analytical conditions, as described below.

\subsection{Individual Fragments: Analytical Techniques}

We measured three oxygen isotopes in the individually-mounted particles (Section 3.1) using the University of Hawai'i Cameca ims 1280 ion microprobe in multicollection mode. A 25-30 $\mathrm{pA} \mathrm{Cs}^{+}$primary ion beam was focused to $\sim 2 \mu \mathrm{m}$ to allow for analysis of small phases in these particles. An electron flood gun was used for charge compensation. The detector configuration is given in Table 2.

Each measurement spot was presputtered for 3 minutes and then measured for 10 minutes (30 cycles, 20 seconds per cycle). The ${ }^{16} \mathrm{O}$ count rate for olivine was $\sim 3 \times 10^{7}$ cps. The 


\begin{tabular}{lccc}
\hline Isotope & Detector & Mass-Resolving Power & Notes \\
\hline${ }^{16} \mathrm{O}$ & L1 FC & $\sim 2000$ & $10^{11} \Omega$ resistor \\
${ }^{17} \mathrm{O}$ & Monocollection EM & $\sim 5500$ & ${ }^{16} \mathrm{OH}^{-}$resolved from ${ }^{17} \mathrm{O}^{-}$ \\
${ }^{18} \mathrm{O}$ & H2 EM & $\sim 2000$ & Exit Slit \#1 $(500 \mu \mathrm{m})$ \\
\hline
\end{tabular}

Table 2: Detector configurations (UH Cameca ims 1280 ion probe) for measurements of individual comet Wild 2 fragments. $\mathrm{EM}=$ electron multiplier, $\mathrm{FC}=$ Faraday cup.

data was corrected for background, deadtime, and interference from ${ }^{16} \mathrm{OH}^{-}$to ${ }^{17} \mathrm{O}^{-}$(typically $<0.2 \%)$. Variable yields between the Mono EM $\left({ }^{17} \mathrm{O}\right)$ and $\mathrm{H} 2 \mathrm{EM}\left({ }^{18} \mathrm{O}\right)$ as well as mass-dependent isotope fractionation between the measured and true value of the terrestrial standards were simultaneously corrected by dividing the measured EM counts by a yield factor. The yield was simply the error-weighted mean of measured $\mathrm{O}$ isotope ratios $\left({ }^{17} \mathrm{O} /{ }^{16} \mathrm{O}\right.$ for the Mono EM, ${ }^{18} \mathrm{O} /{ }^{16} \mathrm{O}$ for the $\mathrm{H} 2 \mathrm{EM}$ ) of the standard divided by the true ratio of the standard.

The measured compositions of each mineral phase were corrected for instrumental mass fractionation by comparing with a set of measurements of appropriate mineral standards of known composition, made before and after the unknown (there was no significant drift in the standard measurements over time). If there were no appropriate standards available, we used San Carlos olivine to standardize the measurements: Caligula (amorphous silicate), Tintin (amorphous ferromagnesian silicate), and the mesostasis of Iris were standardized with San Carlos olivine. The magnitude of unaccounted instrumental mass fractionation for these measurements is likely no more than a few \%o, as their chemical compositions do not differ dramatically from olivine (e.g., Jogo et al., 2012),except for Tintin which is discussed in Section 7.1.

After SIMS measurements, we made sure we cleanly measured our intended targets by imaging the Stardust particles by secondary electron microscopy (SEM). We discarded any measurement spots that did not cleanly hit a single phase or that overlapped with the epoxy embedding medium, except for our measurement of Pooka. The measurement of this small particle overlapped marginally with the surrounding epoxy, and there was not enough area left to make another measurement. The epoxy has an isotopically light $\mathrm{O}$ composition on 
the TFL $\left(\delta^{18} \mathrm{O} \approx-85 \%, \delta^{17} \mathrm{O} \approx-45 \%\right.$, measured relative to a known mineral standard by ion probe) and ${ }^{16} \mathrm{O}$ count rate that is $\sim 1 \%$ of enstatite or olivine. We corrected Pooka's measured $\mathrm{O}$ isotope composition using a simple mixing calculation:

$$
\left(\frac{{ }^{j} \mathrm{O}}{{ }^{16} \mathrm{O}}\right)_{\text {true }}=\left(\frac{{ }^{j} \mathrm{O}}{{ }^{16} \mathrm{O}}\right)_{\text {meas. }}+(X)\left(\frac{1-F}{F}\right)\left(\left(\frac{{ }^{j} \mathrm{O}}{{ }^{16} \mathrm{O}}\right)_{\text {meas. }}-\left(\frac{{ }^{j} \mathrm{O}}{{ }^{16} \mathrm{O}}\right)_{\text {epoxy }}\right)
$$

where $X$ is the ${ }^{16} \mathrm{O}$ count rate in the epoxy divided by the count rate in the standard $\left(\mathrm{C}_{\text {epoxy }} / \mathrm{C}_{\text {stand. }}\right), j$ is the numerator isotope (i.e., 17 or 18$)$, and $F$ is the volume fraction of the contaminated spot which was enstatite $(1-F$ was epoxy). It is possible to estimate $\mathrm{F}$ from the ${ }^{16} \mathrm{O}$ count rates:

$$
F=\frac{\mathrm{C}_{\text {Pooka }}-\mathrm{C}_{\text {stand. }}}{\mathrm{C}_{\text {epoxy }}-\mathrm{C}_{\text {stand }}}
$$

For Pooka, this correction resulted in a $+4.4 \%$ shift in $\delta^{18} \mathrm{O}$ and $+2.3 \%$ shift in $\delta^{17} \mathrm{O}$. The epoxy also affected the measurement by contributing a very large amount of ${ }^{16} \mathrm{OH}^{-}$to ${ }^{17} \mathrm{O}^{-}$. We measured the ${ }^{16} \mathrm{OH}^{-}$count rate after the measurement, and made a conservative assumption that the contribution to ${ }^{17} \mathrm{O}^{-}$had a $1 \sigma$ uncertainty of $5 \mathrm{ppm}$ (mean value $=15$ $\mathrm{ppm}$ ). This correction resulted in a shift of $-2.4 \%$ in $\delta^{17} \mathrm{O}$ for Pooka. All other measurements had much smaller ${ }^{16} \mathrm{OH}^{-}$corrections $(<0.2 \%)$, making the correction uncertainty negligible compared to the statistical and other systematic uncertainties.

\subsection{Bulb of Track 74: Analytical Techniques}

Using a $<3 \mathrm{pA}$ primary $\mathrm{Cs}^{+}$beam on the University of Hawai'i Cameca ims 1280 ion probe focused to $\sim 500 \mathrm{~nm}$, we acquired $128 \times 128$ pixel, $15 \mu \mathrm{m} \times 15 \mu \mathrm{m}$ scanning ion maps for a total of $\sim 15$ hours per map. In each $\sim 75$ s measurement cycle, we collected ${ }^{16} \mathrm{O}^{-}$, ${ }^{17} \mathrm{O}^{-},{ }^{18} \mathrm{O}^{-}$simultaneously on three electron multipliers (L1, Monocollection, H2) and then peak-jumped to ${ }^{28} \mathrm{Si}^{-},{ }^{27} \mathrm{Al},{ }^{24} \mathrm{Mg}^{16} \mathrm{O}^{-}$, and ${ }^{56} \mathrm{Fe}$ (all on Monocollection EM). For two maps (\#4 and \#5) we collected ${ }^{27} \mathrm{Al}^{16} \mathrm{O}^{-}$and ${ }^{56} \mathrm{Fe}^{16} \mathrm{O}^{-}$instead of ${ }^{27} \mathrm{Al}^{-}$and ${ }^{56} \mathrm{Fe}^{-}$(because the oxides had a larger seconary ion yield) and also collected ${ }^{12} \mathrm{C}^{-}$and ${ }^{13} \mathrm{C}^{-}$(simultaneously) to search for presolar silicon carbide grains. The mass resolving power for ${ }^{17} \mathrm{O}^{-}$was $\sim 5500$ to 

cometary particles from the surrounding aerogel.
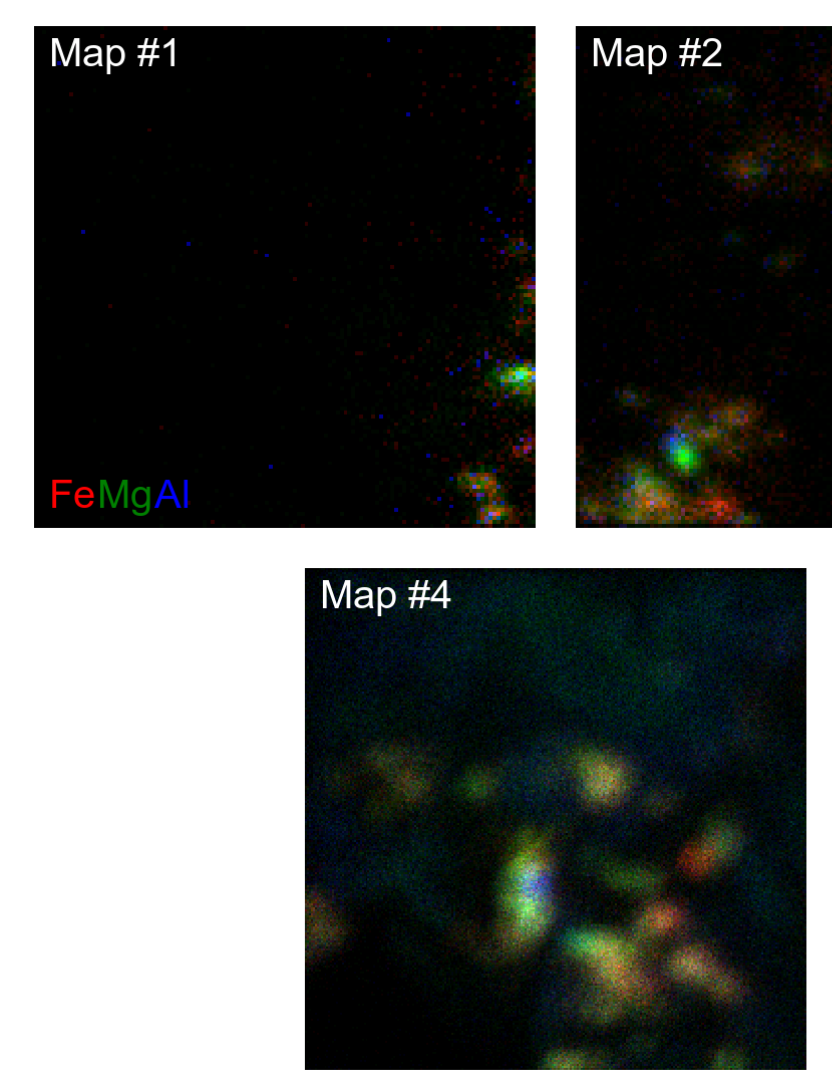
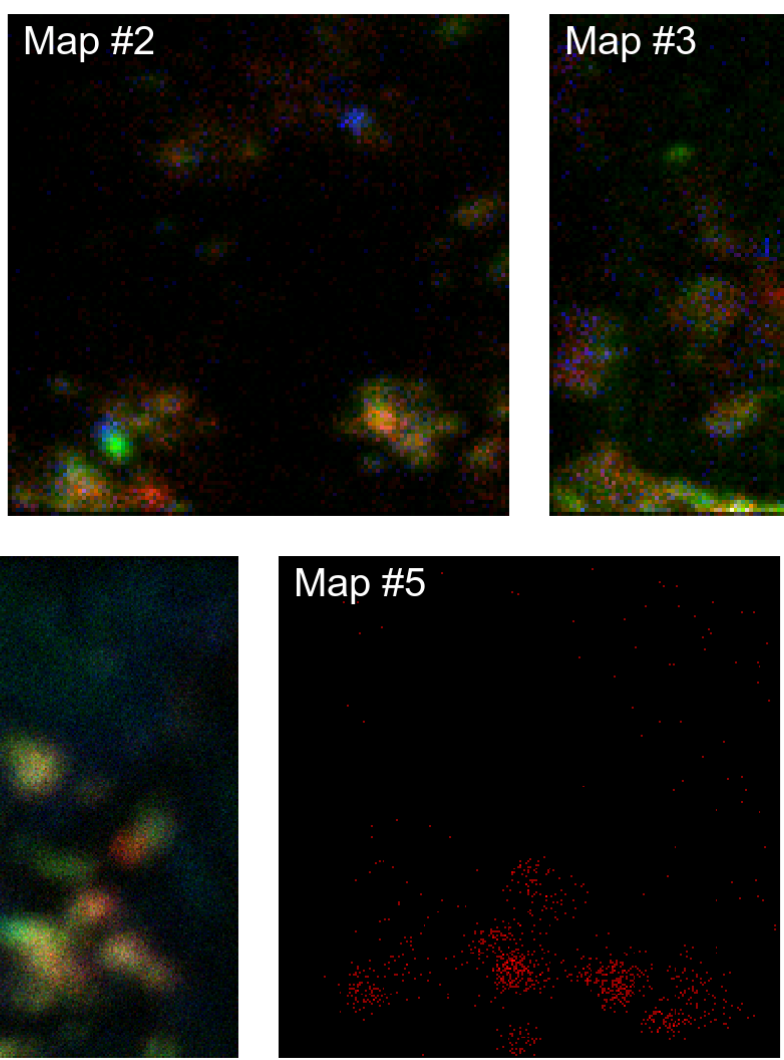

Figure 6: Scanning ion imaging maps of the five regions measured in the bulb of Stardust track C2052,74 measurements. Each map is $15 \times 15 \mu \mathrm{m} .{ }^{56} \mathrm{Fe}^{16} \mathrm{O}$ is red, ${ }^{24} \mathrm{Mg}^{16} \mathrm{O}$ is green, ${ }^{27} \mathrm{Al}^{16} \mathrm{O}$ is blue. The mass peaks for ${ }^{24} \mathrm{Mg}^{16} \mathrm{O}$ and ${ }^{27} \mathrm{Al}^{16} \mathrm{O}$ were lost during the measurement of Map \#5.

minimize the contribution of the ${ }^{16} \mathrm{OH}^{-}$interference on ${ }^{17} \mathrm{O}^{-}$. The electron flood gun was used for charge compensation. We registered the maps collected for each cycle using the ${ }^{16} \mathrm{O}^{-}$channel to account for position drift during the long measurement.

The goal of this measurement was to determine the $\mathrm{O}$ composition of the cometary (or standard) material relative to the surrounding aerogel, which has a known O isotopic composition, $\delta^{18} \mathrm{O} \approx-1.1 \%$ and $\delta^{17} \mathrm{O} \approx-0.5 \%$ (McKeegan et al., 2006). The cometary material (or standard) contains $\mathrm{Mg}, \mathrm{Al}$, or Fe at a much higher concentration than the aerogel. From our collected $\mathrm{MgO}, \mathrm{AlO}$, and $\mathrm{FeO}$ maps (Figure 6) we were easily able to distinguish (using a simple thresholding algorithm or drawing by eye) spatially and compositionally distinct
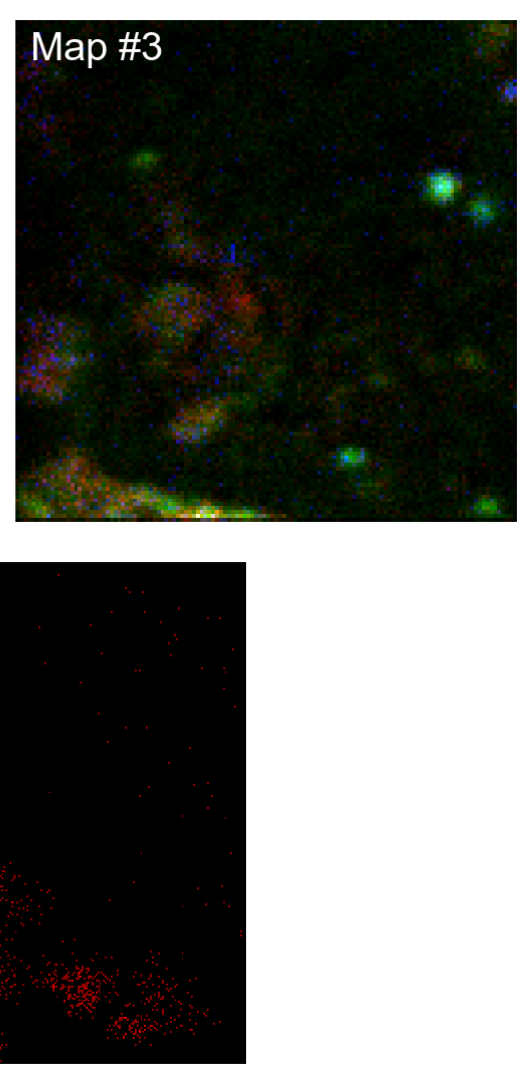
for $\mathrm{Mg}$ (

We calculated ${ }^{18} \mathrm{O} /{ }^{16} \mathrm{O}$ and ${ }^{17} \mathrm{O} /{ }^{16} \mathrm{O}$ in the identified individual cometary particles. We normalized this composition to the surrounding aerogel O composition and calculated un- 
certainties by the following Monte Carlo method. For each region of interest consisting of $N$ cometary pixels, we randomly selected $N$ background aerogel pixels and calculated the ${ }^{18} \mathrm{O} /{ }^{16} \mathrm{O}$ and ${ }^{17} \mathrm{O} /{ }^{16} \mathrm{O}$ ratios in this set of aerogel pixels. We calculated $\delta^{18} \mathrm{O}$ and $\delta^{17} \mathrm{O}$ for the region of interest relative to these aerogel ratios (while accounting for the known $\mathrm{O}$ composition of the aerogel: $\delta^{18} \mathrm{O}=-1.09, \delta^{17} \mathrm{O}=-0.52$ (McKeegan et al., 2006)), and repeated the process $10^{5}$ times. From this distribution, we computed a mean and standard deviation which correspond to the $\delta^{18} \mathrm{O}$ and $\delta^{17} \mathrm{O}$ values and uncertainties for the cometary region. We also computed these values and uncertainties by selecting connected groups of $N$ aerogel pixels, instead of random unconnected pixels. The $\delta^{18} \mathrm{O}$ and $\delta^{17} \mathrm{O}$ values were very similar in both analyses, but the uncertainties in the unconnected pixel analysis were larger. We chose to use the unconnected pixel analysis because it yielded more conservative errors. Analyzing a mixture of cometary material and aerogel would move the measured value closer to $\delta^{17,18} \mathrm{O}=(0,0)$. Each region of interest was chosen to minimize the contribution of surrounding aerogel.

\subsection{SEM, EPMA, FIB, TEM}

We used two SEMs with EDS for sample preparation and characterization: a JEOL 5900LV SEM (University of Hawai'i at Mānoa) with a Noran EDS system, and a Tescan Vega 3 with an Oxford EDS system (University of California, Berkeley). We used a JEOL JXA-8500F Electron Microprobe at the University of Hawai'i at Mānoa to acquire elemental maps in compressed aerogel from the bulb of track 74 . The operating voltage was $15 \mathrm{keV}$, and we acquired maps of 15 elements (including $\mathrm{Mg}, \mathrm{Fe}, \mathrm{O}, \mathrm{Si}, \mathrm{In}$ ) alternating on five wavelength-dispersive spectrometers.

We used a focused ion beam (FIB) instrument and a transmission electron microscope (TEM) to extract and analyze particles in compressed aerogel from the bulb of track 74 . Both FIB and TEM were housed at the National Center for Electron Microscopy at Lawrence Berkeley National Laboratory. The FIB was an FEI Strata 235 Dual Beam and the TEM was a Zeiss Libra $200 \mathrm{MC}$ operating at $200 \mathrm{keV}$. We used the TEM for energy-filtered imaging and electron diffraction. 


\section{Results of Standard Measurements}

To assess the accuracy and precision of our ion probe measurements, we repeatedly measured standards of known O isotopic composition. Standards for individual fragments (Section 2.1) and Track 74 bulb particles (Section 2.2) were measured differently, as described below.

\subsection{Individual Fragments: Results of Standard Measurements}

We measured a San Carlos olivine standard analog before and after our measurement of each particle of unknown $\mathrm{O}$ isotopic composition. This analog mount was prepared similar to the Stardust sample: a piece of San Carlos olivine was embedded in the tip of an epoxy bullet, microtomed, and mounted as described in Section 3.1. The specifics of our sample preparation procedure evolved as we improved methods to, e.g., control the height of the epoxy bullet in the mount. For this reason, we used a contemporaneously prepared olivine analog for each measurement of individual Stardust particles. By comparing the measured composition of the center-mounted San Carlos olivine with the measured value of San Carlos olivine in the standard buckler, we could measure any systematic instrumental mass fractionation between the center-mounted particle and the buckler standard. Such a shift can be caused by slightly different sample topography between the center and buckler. Sample topography can significantly affect instrumental mass fractionation (Kita et al., 2009). For most of the measurements, this center-buckler shift is consistent with $0 \%$ per amu, and is at most $1.5 \%$ per amu. We constrained the center-buckler shift by measuring $5-10$ spots on both the buckler and center of the San Carlos olivine analog sample before and after each unknown measurement. The different values between sessions likely reflect changes in sample topography from an evolving mounting procedure, so we correct each unknown value with the center-buckler shift in the contemporaneously mounted olivine standard, measured before and after the Stardust particle. There was no significant instrumental mass fractionation as a function of position within the center-mounted San Carlos olivine. 


\subsection{Bulb of Track 74: Results of Standard Measurements}

We measured Allende spinels pressed into aerogel (Section 3.2) to have O isotopic composition consistent with that measured for individual spinel grains distributed onto Au foil (Makide et al., 2009a), except for two outliers (see Figure 7). The two outliers measurements have cation/oxygen ratios (as measured by scanning ion imaging in the ion probe) different from the other spots, indicating that they are non-spinel contaminants in the acid residue from Allende that were pressed alongside spinel grains into the aerogel. The precision of the pressed-aerogel spinel measurements was similar to that of spinels measured on $\mathrm{Au}$ foil. We measured the $\mathrm{O}$ isotopic composition of olivine shot into aerogel (Section 3.2) to be consistent with its actual composition, though with large uncertainties due to the small size of fragments we measured. Matrix effects from analyzing minerals in aerogel are constrained to be small compared to the characteristic uncertainties of the measurements.

In order to estimate the $\mathrm{Al} / \mathrm{O}, \mathrm{Mg} / \mathrm{O}$, and $\mathrm{Fe} / \mathrm{O}$ atom ratio of the Stardust fragments from ion probe measurements, we must constrain the relative sensitivity of these elements in the ion probe. It is well-known that different elements are detected with different efficiencies in the ion probe. The relative sensitivity factor (RSF) for two elements is defined as the ion probe elemental ratio divided by the "true" elemental ratio (the latter is typically measured by electron-probe microanalyzer (EPMA)). The RSF depends on a number of variables such as the primary ion species and the chemical composition of the substrate (e.g. Bottazzi et al., 1992). We measured an oxide (chromite) and silicate (pyroxene) of known chemical compositions using analytical conditions similar to our Stardust measurements. The mineralogy of the fragments we measure in compressed aerogel from the bulb of Track 74 is not known. However, the majority of the O-bearing fragments are likely silicates or oxides (Stodolna et al., 2012). The RSF can vary significantly between minerals (up to $\sim 2 \times$ ), so these estimates of $\mathrm{Al} / \mathrm{O}, \mathrm{Mg} / \mathrm{O}$, and $\mathrm{Fe} / \mathrm{O}$ in the Stardust fragments have large uncertainties. The RSFs of $\mathrm{Al} / \mathrm{O}, \mathrm{Mg} / \mathrm{O}$, and $\mathrm{Fe} / \mathrm{O}$ as calculated from ion probe and EPMA measurements of pyroxene and chromite standards, that are used in our estimates of these ratios in our Stardust measurements (Table 5), are given in Table 3. 


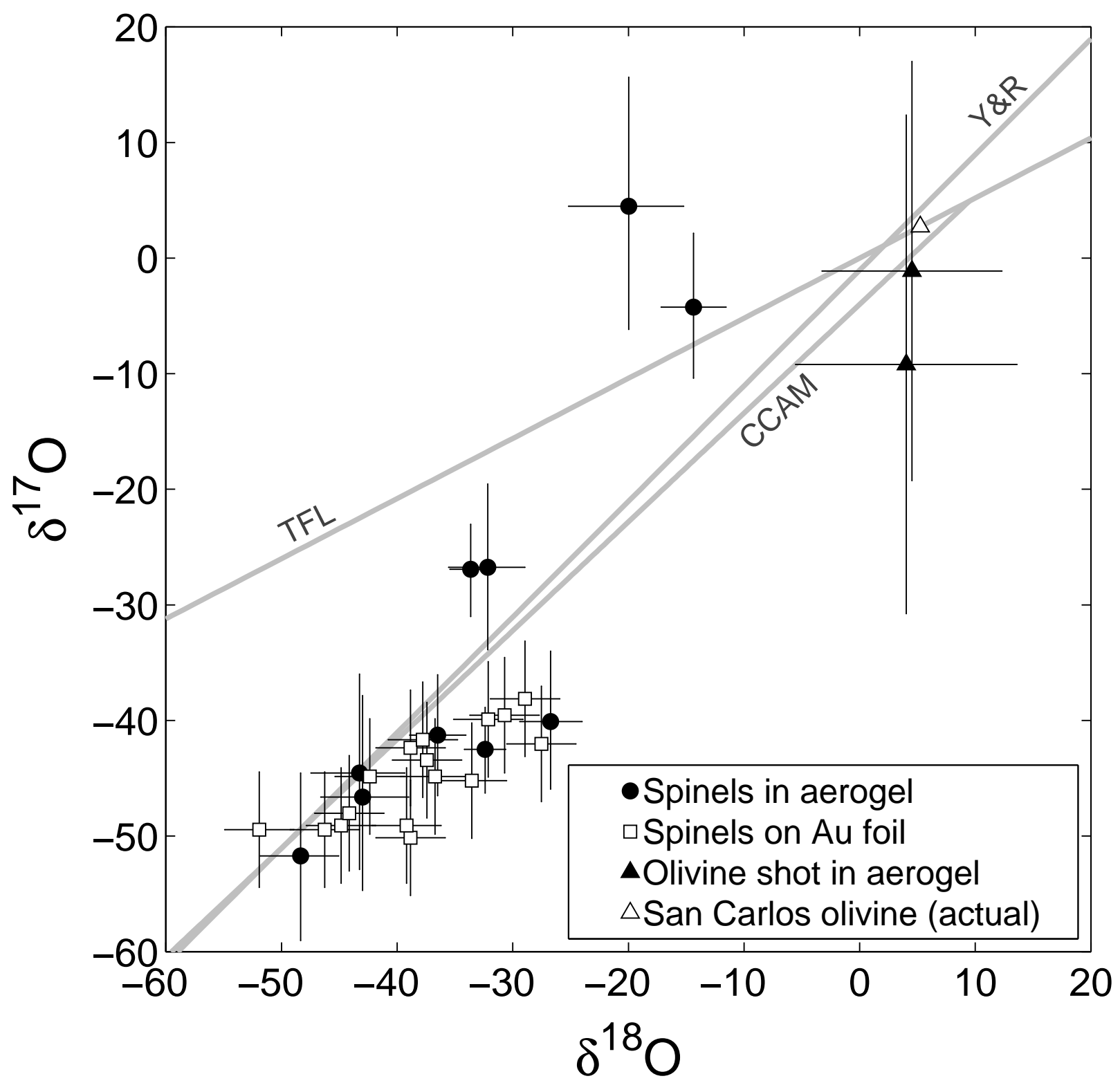

Figure 7: Oxygen 3-isotope plot showing Allende spinel separates pressed into aerogel (black circles, this study), individual spinels measured on Au foil (open squares, Makide et al. (2009a)), olivine shot into aerogel (black triangles, this study), and the true value of San Carlos olivine (open traingle). Uncertainties are $2 \sigma$. The terrestrial fraction line (TFL), carbonaceous chondrite anhydrous mineral line (CCAM, Clayton et al. (1977)) and Young \& Russell line (Y\&R, Young and Russell (1998)) are indicated. 


\begin{tabular}{lcc}
\hline Species Ratio & RSF $_{\text {Pyroxene }}$ & RSF $_{\text {Chromite }}$ \\
\hline${ }^{27} \mathrm{Al} /{ }^{16} \mathrm{O}$ & 0.015 & $6.1 \times 10^{-3}$ \\
${ }^{27} \mathrm{Al}^{16} \mathrm{O} /{ }^{16} \mathrm{O}$ & 0.40 & 0.37 \\
${ }^{24} \mathrm{Mg}^{16} \mathrm{O} /{ }^{16} \mathrm{O}$ & 0.029 & 0.031 \\
${ }^{56} \mathrm{Fe} /{ }^{16} \mathrm{O}$ & $6.0 \times 10^{-3}$ & $4.0 \times 10^{-3}$ \\
${ }^{56} \mathrm{Fe}{ }^{16} \mathrm{O} /{ }^{16} \mathrm{O}$ & 0.024 & 0.045 \\
\hline
\end{tabular}

Table 3: Relative sensitivity factors in pyroxene and chromite.

\section{O Isotopic Compositions of comet Wild 2 Particles}

Our measurements of the extracted Stardust fragments are shown in Figure 8 and listed in Table 4. Most of the measurements of the extracted $>2 \mu \mathrm{m}$ fragments are consistent with the terrestrial fractionation line.

Our O isotope measurements of the fine-grained material in the bulb of Stardust track C2052,74 are listed in Table 5 and plotted in Figure 9. The chemical composition ( $\mathrm{Mg}, \mathrm{Al}$, and Fe relative to $\mathrm{O}$ ) of these particles is also given in Table 5. The Wild 2 fine-grained material shows a very diverse range of $\mathrm{O}$ isotope compositions. 


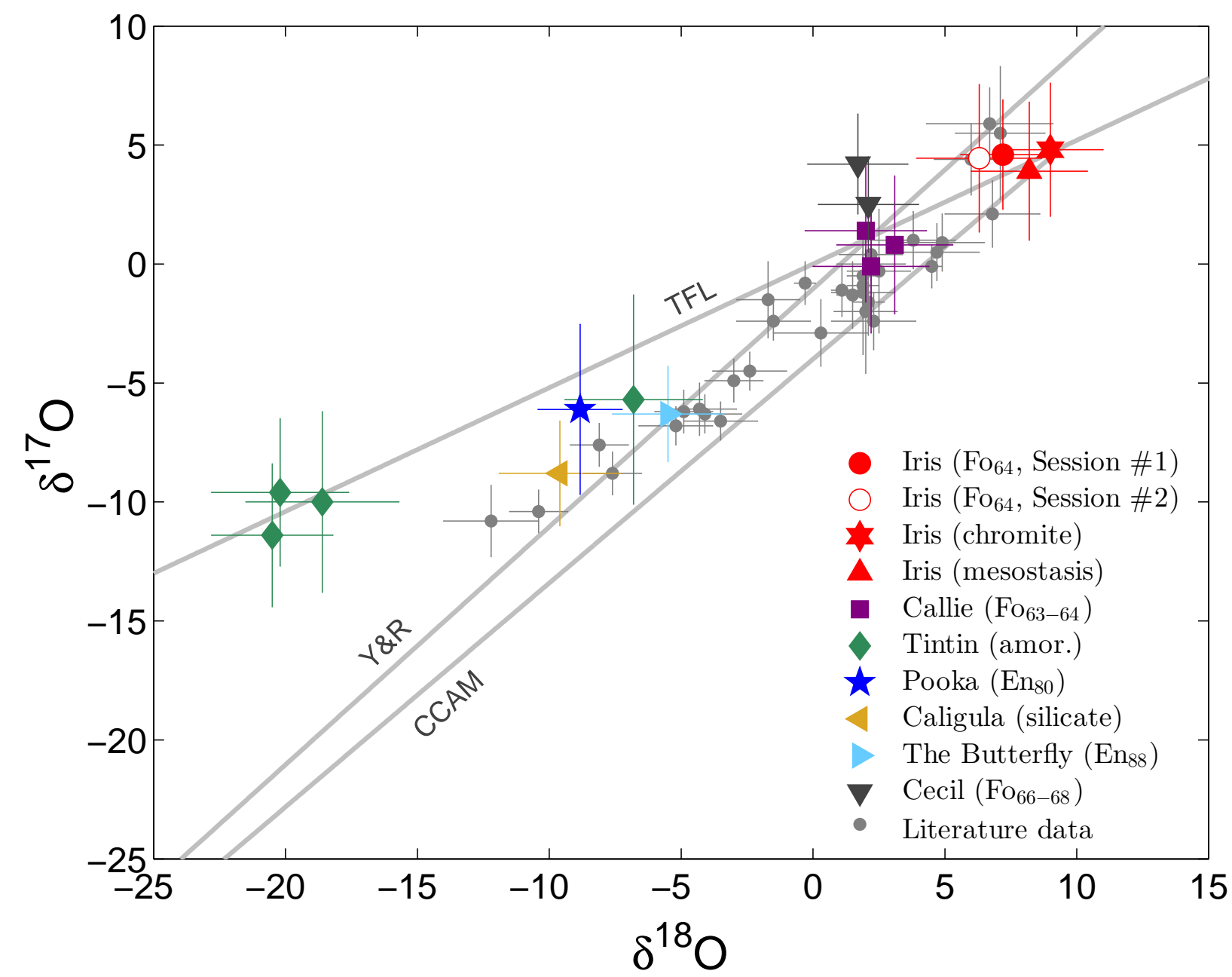

Figure 8: Oxygen 3-isotope plot showing the compositions of the seven extracted Wild 2 fragments. Iris olivine was measured in two separate sessions to verify our measurement reproducibility (Section 6.1.1). Literature data are from McKeegan et al. (2006); Nakamura et al. (2008); Nakashima et al. (2012a); eight measurements of ${ }^{16} \mathrm{O}$-rich particles from these are not shown. Uncertainties are $2 \sigma$. 


\begin{tabular}{|c|c|c|c|c|c|c|c|}
\hline Name & Size $(\mu \mathrm{m})$ & Track & Location & Phase & $\delta^{18} \mathrm{O}(\% 0)$ & $\delta^{17} \mathrm{O}\left(\%_{00}\right)$ & $\Delta^{17} \mathrm{O}(\%)$ \\
\hline $\operatorname{Iris}^{\dagger}$ & $23 \times 10$ & $\mathrm{C} 2052,74$ & Terminal & $\begin{array}{l}\mathrm{Fo}_{64}\left(1^{\ddagger}\right) \\
\mathrm{Fo}_{64}\left(2^{\ddagger}\right) \\
\text { chromite } \\
\text { mesostasis }\end{array}$ & $\begin{array}{l}7.2 \pm 1.6 \\
6.3 \pm 2.4 \\
9.0 \pm 2.0 \\
8.2 \pm 2.2\end{array}$ & $\begin{array}{l}4.6 \pm 2.3 \\
4.4 \pm 3.1 \\
4.8 \pm 2.8 \\
3.9 \pm 2.9\end{array}$ & $\begin{array}{r}0.9 \pm 2.4 \\
1.2 \pm 3.3 \\
0.1 \pm 3.0 \\
-0.4 \pm 3.1\end{array}$ \\
\hline Callie & $10 \times 6$ & $\mathrm{C} 2052,74$ & Terminal & $\begin{array}{c}\mathrm{Fo}_{64}(1) \\
\mathrm{Fo}_{64}(2) \\
\mathrm{Fo}_{63} \\
\text { average }\end{array}$ & $\begin{array}{l}3.1 \pm 2.2 \\
2.2 \pm 2.2 \\
2.0 \pm 2.3 \\
2.4 \pm 1.3\end{array}$ & $\begin{array}{r}0.8 \pm 2.9 \\
-0.1 \pm 2.8 \\
1.4 \pm 3.0 \\
0.7 \pm 1.7\end{array}$ & $\begin{array}{r}-0.8 \pm 3.1 \\
-1.3 \pm 3.0 \\
0.4 \pm 3.2 \\
-0.6 \pm 1.8\end{array}$ \\
\hline Tintin & $10 \times 8$ & C2052,74 & Bulb & $\begin{array}{l}\text { amor. (1) } \\
\text { amor. (2) } \\
\text { amor. (3) } \\
\text { amor. (4) }\end{array}$ & $\begin{array}{r}-18.6 \pm 2.9 \\
-20.2 \pm 2.6 \\
-6.8 \pm 2.6 \\
-20.5 \pm 2.3\end{array}$ & $\begin{array}{r}-10.0 \pm 3.8 \\
-9.6 \pm 3.1 \\
-5.7 \pm 4.4 \\
-11.4 \pm 3.0\end{array}$ & $\begin{array}{r}-0.3 \pm 4.1 \\
0.9 \pm 3.3 \\
-2.1 \pm 4.6 \\
-0.7 \pm 3.2\end{array}$ \\
\hline Pooka & $3 \times 3$ & C2061,113 & Terminal & $\operatorname{En}_{80}$ & $-8.8 \pm 1.6$ & $-6.1 \pm 3.6$ & $-1.5 \pm 3.7$ \\
\hline Caligula & $12 \times 6$ & C2035,105 & Terminal & silicate & $-9.6 \pm 2.3$ & $-8.8 \pm 2.2$ & $-3.8 \pm 2.5$ \\
\hline The Butterfly & $3 \times 2$ & C2009,77 & Bulb & $\operatorname{En}_{88}$ & $-5.5 \pm 2.1$ & $-6.3 \pm 2.0$ & $-3.4 \pm 2.2$ \\
\hline Cecil & $10 \times 8$ & C2062,162 & Terminal & $\begin{array}{c}\mathrm{Fo}_{66} \\
\mathrm{Fo}_{68} \\
\text { average }\end{array}$ & $\begin{array}{l}1.7 \pm 1.9 \\
2.1 \pm 1.9 \\
1.9 \pm 1.3\end{array}$ & $\begin{array}{l}4.2 \pm 2.1 \\
2.5 \pm 2.0 \\
3.4 \pm 1.4\end{array}$ & $\begin{array}{l}3.4 \pm 2.3 \\
1.4 \pm 2.2 \\
2.4 \pm 1.6\end{array}$ \\
\hline
\end{tabular}

Table 4: O isotope compositions of individual Stardust cometary particles. Parentheses indicate multiple measurement spots on the same grain. Errors are $2 \sigma$. "amor." = amorphous. ${ }^{\ddagger}$ Iris olivine was measured in two separate sessions to verify our measurement reproducibility (Section 6.1.1). ${ }^{\dagger}$ These measurements were reported previously in Ogliore et al. (2012). 


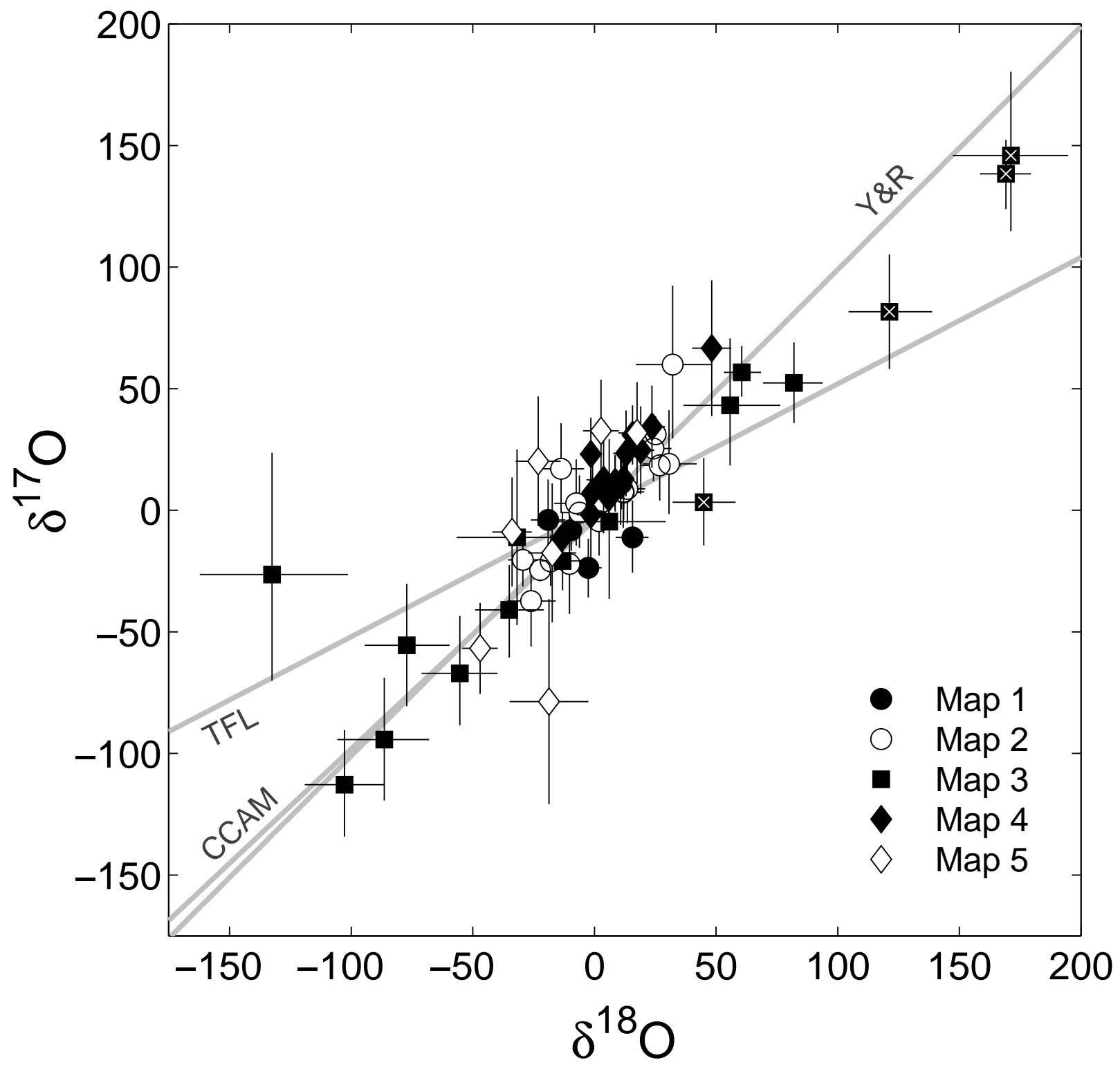

Figure 9: Oxygen 3-isotope plot showing the compositions of 63 particles in the bulb of Stardust track C2052,74. The terrestrial fraction line (TFL), carbonaceous chondrite anhydrous mineral line (CCAM) and Young \& Russell line (Y\&R) are indicated. Remeasured particles (Section 6.1.2) are marked with a white $\mathrm{X}$ inside a black square. Uncertainties are $2 \sigma$. 


\begin{tabular}{|c|c|c|c|c|c|c|c|c|}
\hline Particle & Map & Size $(\mu \mathrm{m})$ & $\mathrm{Mg} / \mathrm{O}$ & $\mathrm{Al} / \mathrm{O}$ & $\mathrm{Fe} / \mathrm{O}$ & $\delta^{18} \mathrm{O}(\% 0)$ & $\delta^{17} \mathrm{O}(\%)$ & $\Delta^{17} \mathrm{O}(\%)$ \\
\hline 1 & 1 & 0.7 & 0.02 & $<0.01$ & 0.01 & $15.7 \pm 6.5$ & $-11.1 \pm 14.6$ & $-19.3 \pm 15.0$ \\
\hline 2 & 1 & 0.9 & 0.02 & $<0.01$ & 0.02 & $-2.6 \pm 5.3$ & $-23.7 \pm 11.9$ & $-22.3 \pm 12.2$ \\
\hline 3 & 1 & 1.3 & 0.09 & $<0.01$ & 0.03 & $-9.6 \pm 3.6$ & $-8.1 \pm 8.7$ & $-3.1 \pm 8.9$ \\
\hline 4 & 1 & 0.6 & 0.02 & $<0.01$ & 0.05 & $-19.1 \pm 6.9$ & $-3.9 \pm 16.2$ & $6.0 \pm 16.6$ \\
\hline 5 & 1 & 1.5 & 0.04 & $<0.01$ & 0.04 & $-1.6 \pm 3.2$ & $4.6 \pm 7.5$ & $5.5 \pm 7.7$ \\
\hline 6 & 2 & 1.0 & 0.07 & $<0.01$ & 0.02 & $1.9 \pm 7.3$ & $-4.5 \pm 14.2$ & $-5.5 \pm 15.0$ \\
\hline 7 & 2 & 2.8 & 0.04 & $<0.01$ & 0.06 & $9.3 \pm 2.6$ & $11.5 \pm 4.9$ & $6.7 \pm 12.2$ \\
\hline 8 & 2 & 1.0 & 0.06 & $<0.01$ & 0.04 & $13.5 \pm 7.3$ & $8.8 \pm 13.9$ & $1.7 \pm 8.9$ \\
\hline 9 & 2 & 0.9 & 0.17 & 0.06 & 0.08 & $11.8 \pm 8.0$ & $7.6 \pm 15.2$ & $1.4 \pm 16.6$ \\
\hline 10 & 2 & 0.9 & 0.05 & $<0.01$ & 0.05 & $26.9 \pm 8.2$ & $18.5 \pm 14.6$ & $4.5 \pm 7.7$ \\
\hline 11 & 2 & 1.3 & 0.06 & $<0.01$ & 0.06 & $11.2 \pm 5.5$ & $11.2 \pm 10.6$ & $5.4 \pm 14.7$ \\
\hline 12 & 2 & 1.2 & 0.07 & $<0.01$ & 0.06 & $-29.4 \pm 5.9$ & $-20.3 \pm 10.8$ & $-5.1 \pm 5.1$ \\
\hline 13 & 2 & 0.7 & 0.03 & $<0.01$ & 0.01 & $30.7 \pm 10.9$ & $19.1 \pm 21.2$ & $3.2 \pm 14.4$ \\
\hline 14 & 2 & 0.7 & 0.02 & $<0.01$ & 0.01 & $-26.1 \pm 9.8$ & $-37.3 \pm 18.6$ & $-23.8 \pm 15.8$ \\
\hline 15 & 2 & 0.8 & 0.04 & $<0.01$ & 0.03 & $-7.5 \pm 8.7$ & $2.9 \pm 17.5$ & $6.7 \pm 15.2$ \\
\hline 16 & 2 & 0.8 & 0.06 & $<0.01$ & 0.03 & $-13.8 \pm 9.1$ & $17.1 \pm 18.3$ & $24.2 \pm 11.0$ \\
\hline 17 & 2 & 0.6 & 0.02 & $<0.01$ & 0.02 & $-10.3 \pm 11.0$ & $-22.1 \pm 20.7$ & $-16.7 \pm 11.2$ \\
\hline 18 & 2 & 3.0 & 0.11 & $<0.01$ & 0.09 & $-22.3 \pm 2.2$ & $-24.5 \pm 4.4$ & $-12.9 \pm 21.9$ \\
\hline 19 & 2 & 1.0 & 0.05 & $<0.01$ & 0.03 & $24.2 \pm 7.2$ & $25.4 \pm 13.6$ & $12.8 \pm 19.2$ \\
\hline 20 & 2 & 1.0 & 0.07 & $<0.01$ & 0.05 & $-6.2 \pm 7.4$ & $-1.0 \pm 14.7$ & $2.3 \pm 18.1$ \\
\hline 21 & 2 & 1.9 & 0.09 & $<0.01$ & 0.09 & $25.1 \pm 4.0$ & $31.3 \pm 7.2$ & $18.2 \pm 18.9$ \\
\hline 22 & 2 & 1.2 & 0.32 & 0.02 & 0.05 & $12.0 \pm 6.1$ & $7.3 \pm 11.2$ & $1.0 \pm 21.5$ \\
\hline 23 & 2 & 1.3 & 0.07 & $<0.01$ & 0.07 & $-18.0 \pm 5.0$ & $-21.0 \pm 10.2$ & $-11.6 \pm 4.5$ \\
\hline 24 & 2 & 2.0 & 0.18 & 0.01 & 0.13 & $3.0 \pm 3.4$ & $3.4 \pm 6.8$ & $1.8 \pm 14.1$ \\
\hline 25 & 2 & 1.2 & 0.08 & $<0.01$ & 0.14 & $8.6 \pm 5.7$ & $10.9 \pm 11.2$ & $6.4 \pm 15.2$ \\
\hline 26 & 2 & 0.5 & 0.09 & $<0.01$ & 0.10 & $32.2 \pm 15.4$ & $59.9 \pm 31.2$ & $43.2 \pm 7.5$ \\
\hline 27 & 3 & 0.9 & 0.06 & $<0.01$ & 0.06 & $-102.8 \pm 16.2$ & $-112.8 \pm 21.6$ & $-59.4 \pm 15.0$ \\
\hline 28 & 3 & 0.5 & 0.11 & 0.03 & 0.11 & $-132.6 \pm 30.2$ & $-26.4 \pm 46.7$ & $42.5 \pm 12.2$ \\
\hline 29 & 3 & 0.7 & 0.07 & $<0.01$ & 0.02 & $6.1 \pm 23.8$ & $-4.6 \pm 32.6$ & $-7.8 \pm 8.9$ \\
\hline
\end{tabular}




\begin{tabular}{|c|c|c|c|c|c|c|c|c|}
\hline 30 & 3 & 1.0 & 0.24 & 0.02 & 0.09 & $-55.3 \pm 15.4$ & $-67.1 \pm 22.2$ & $-38.3 \pm 16.6$ \\
\hline 31 & 3 & 0.8 & 0.13 & 0.01 & 0.02 & $-86.4 \pm 18.6$ & $-94.3 \pm 25.0$ & $-49.3 \pm 7.7$ \\
\hline 32 & 3 & 1.1 & 0.02 & $<0.01$ & 0.09 & $-35.0 \pm 13.9$ & $-41.0 \pm 18.8$ & $-22.8 \pm 14.7$ \\
\hline 33 & 3 & 1.9 & 0.04 & $<0.01$ & 0.06 & $-13.1 \pm 8.5$ & $-20.8 \pm 12.1$ & $-14.0 \pm 5.1$ \\
\hline 34 & 3 & 2.4 & 0.05 & 0.01 & 0.08 & $60.6 \pm 7.4$ & $56.7 \pm 10.2$ & $25.2 \pm 14.4$ \\
\hline 35 & 3 & 0.9 & 0.04 & $<0.01$ & 0.03 & $-77.2 \pm 17.2$ & $-55.5 \pm 24.9$ & $-15.4 \pm 15.8$ \\
\hline 36 & 3 & 1.5 & 0.08 & $<0.01$ & 0.11 & $82.0 \pm 12.0$ & $52.4 \pm 16.3$ & $9.7 \pm 15.2$ \\
\hline 37 & 3 & 0.9 & 0.11 & 0.01 & 0.02 & $55.7 \pm 19.7$ & $43.2 \pm 25.9$ & $14.2 \pm 11.0$ \\
\hline $38^{\dagger}$ & 3 & 1.9 & 0.07 & $<0.01$ & 0.11 & $169.1 \pm 10.2$ & $138.3 \pm 14.0$ & $50.4 \pm 11.2$ \\
\hline $39^{\dagger}$ & 3 & 1.1 & 0.11 & $<0.01$ & 0.13 & $121.2 \pm 17.0$ & $81.7 \pm 23.3$ & $18.7 \pm 21.9$ \\
\hline $40^{\dagger}$ & 3 & 1.3 & 0.17 & $<0.01$ & 0.15 & $45.0 \pm 12.8$ & $3.3 \pm 17.7$ & $-20.0 \pm 19.2$ \\
\hline $41^{\dagger}$ & 3 & 0.8 & 0.13 & $<0.01$ & 0.11 & $171.2 \pm 23.5$ & $145.9 \pm 32.6$ & $56.9 \pm 18.1$ \\
\hline 42 & 3 & 0.6 & 0.15 & $<0.01$ & 0.03 & $-31.8 \pm 24.8$ & $-11.1 \pm 35.9$ & $5.4 \pm 18.9$ \\
\hline 43 & 4 & 1.9 & 0.07 & $<0.01$ & 0.06 & $8.4 \pm 3.4$ & $11.5 \pm 11.2$ & $7.1 \pm 11.3$ \\
\hline 44 & 4 & 1.3 & 0.08 & $<0.01$ & 0.04 & $10.8 \pm 4.7$ & $10.0 \pm 15.9$ & $4.4 \pm 16.0$ \\
\hline 45 & 4 & 1.9 & 0.13 & 0.01 & 0.12 & $5.9 \pm 3.3$ & $5.1 \pm 10.4$ & $2.0 \pm 10.5$ \\
\hline 46 & 4 & 1.7 & 0.10 & $<0.01$ & 0.13 & $15.7 \pm 3.8$ & $31.0 \pm 12.0$ & $22.8 \pm 12.2$ \\
\hline 47 & 4 & 1.3 & 0.13 & $<0.01$ & 0.07 & $19.0 \pm 5.4$ & $24.7 \pm 17.8$ & $14.8 \pm 18.1$ \\
\hline 48 & 4 & 1.4 & 0.11 & $<0.01$ & 0.17 & $-1.5 \pm 4.4$ & $23.1 \pm 14.7$ & $23.9 \pm 14.9$ \\
\hline 49 & 4 & 1.7 & 0.12 & 0.01 & 0.15 & $-0.7 \pm 3.7$ & $7.4 \pm 11.4$ & $7.8 \pm 11.6$ \\
\hline 50 & 4 & 1.1 & 0.18 & 0.01 & 0.08 & $-1.5 \pm 5.6$ & $-1.8 \pm 18.3$ & $-1.0 \pm 18.5$ \\
\hline 51 & 4 & 1.9 & 0.16 & 0.01 & 0.17 & $-13.2 \pm 3.2$ & $-11.2 \pm 10.4$ & $-4.3 \pm 10.6$ \\
\hline 52 & 4 & 1.0 & 0.15 & $<0.01$ & 0.11 & $3.8 \pm 6.9$ & $12.5 \pm 21.8$ & $10.5 \pm 22.1$ \\
\hline 53 & 4 & 0.9 & 0.10 & $<0.01$ & 0.06 & $48.2 \pm 7.8$ & $66.7 \pm 27.7$ & $41.6 \pm 28.0$ \\
\hline 54 & 4 & 1.2 & 0.17 & $<0.01$ & 0.14 & $13.0 \pm 5.1$ & $23.4 \pm 17.4$ & $16.7 \pm 17.6$ \\
\hline 55 & 4 & 1.3 & 0.27 & 0.02 & 0.18 & $23.7 \pm 5.1$ & $34.4 \pm 16.7$ & $22.1 \pm 16.9$ \\
\hline 56 & 4 & 1.7 & 0.23 & 0.01 & 0.17 & $12.0 \pm 3.8$ & $12.9 \pm 12.0$ & $6.7 \pm 12.1$ \\
\hline 57 & 5 & 2.2 & - & - & 0.02 & $-33.9 \pm 7.9$ & $-8.9 \pm 22.2$ & $8.7 \pm 22.5$ \\
\hline 58 & 5 & 1.6 & - & - & 0.02 & $2.7 \pm 7.2$ & $32.7 \pm 20.8$ & $31.3 \pm 21.1$ \\
\hline 59 & 5 & 1.4 & - & - & 0.03 & $-17.3 \pm 9.7$ & $-17.5 \pm 28.3$ & $-8.5 \pm 28.8$ \\
\hline
\end{tabular}




\begin{tabular}{ccccccccc}
60 & 5 & 1.7 & - & - & 0.04 & $17.6 \pm 7.5$ & $31.8 \pm 20.7$ & $22.6 \pm 21.1$ \\
61 & 5 & 1.9 & - & - & 0.05 & $-23.1 \pm 9.1$ & $20.2 \pm 26.5$ & $32.2 \pm 26.9$ \\
62 & 5 & 1.4 & - & - & 0.05 & $-18.7 \pm 16.0$ & $-78.7 \pm 42.0$ & $-69.0 \pm 42.8$ \\
63 & 5 & 1.8 & - & - & 0.01 & $-47.0 \pm 7.0$ & $-56.8 \pm 18.5$ & $-32.3 \pm 18.8$ \\
\hline
\end{tabular}

Table 5: The sizes, $\mathrm{Mg} / \mathrm{O}, \mathrm{Al} / \mathrm{O}, \mathrm{Fe} / \mathrm{O}$ elemental (atomic) ratios, and oxygen isotopic compositions of 63 fragments from the bulb of Stardust track C2052,74. ${ }^{\dagger}$ These particles were removed by FIB and reanalyzed for O isotopes (see Section 6.1.2). 


\subsection{Assessing the Reproducibility of O Isotope Measurements}

The accuracy of ion probe measurements can be reduced by various systematic errors caused by, for example, sample topography or conductivity, changes in the primary beam or secondary ion optics, or variable electron-multiplier efficiency. The magnitude of these systematic errors in ion probe measurements of $\mathrm{O}$ isotopes can be large compared to the \%o-level precision needed to address cosmochemical questions. It is therefore necessary to prove that systematic errors in ion probe measurements of small Stardust particles are small compared to the statistical error of the measurement and can safely be ignored. Many types of systematic errors in ion probe analyses can be quantified by measurements of known and homogenous isotope standards which are prepared and mounted analogous to the unknown. Repeated measurements of standards before and after the unknown can be used to estimate systematic errors caused by, for example, drifts in electronmultiplier efficiencies. Sample topography and location as well as differences in charging between sample and standard, on the other hand, produce unpredictable electric-field variations, which can be significant and cannot be quantified with measurements of the standard during the measurement session. To quantify these systematic errors, it is necessary to measure the same sample in more than one measurement session. However, the small size of Stardust samples and difficult sample preparation makes this challenging.

\subsubsection{Individual Fragments: Reproducibility}

The type II chondrule fragment Iris $(23 \times 10 \times 15 \mu \mathrm{m})$ from Stardust Track C2052,74 was microtomed ( 100 nm thick slices), and the potted butt was remounted for SIMS analysis following the initial analyses (Ogliore et al., 2012). We measured the same olivine grains for O isotopes using analytical conditions similar to the first measurement (Section 4.1). We corrected our measurement for any shift seen between the center and ring in an analogously prepared mount of San Carlos olivine. The microtomed faces of Iris before the first and second isotope measurement are shown in Figure 10. The two faces have the same mineral assemblages with similar chemical compositions. Type II chondrules (e.g. Jones, 1990) can contain relict grains with chemical and isotopic compositions distinct from their host phenocrysts. No such grains appeared after microtoming further into Iris, so the $\mathrm{O}$ isotopic composition of the Iris olivine grains should be the same at these two different depths.

The first $\mathrm{O}$ isotope measurement of an Iris olivine was $\delta^{17} \mathrm{O}=4.6 \pm 2.3 \%$, $\delta^{18} \mathrm{O}=7.2 \pm 1.6 \%$ o 


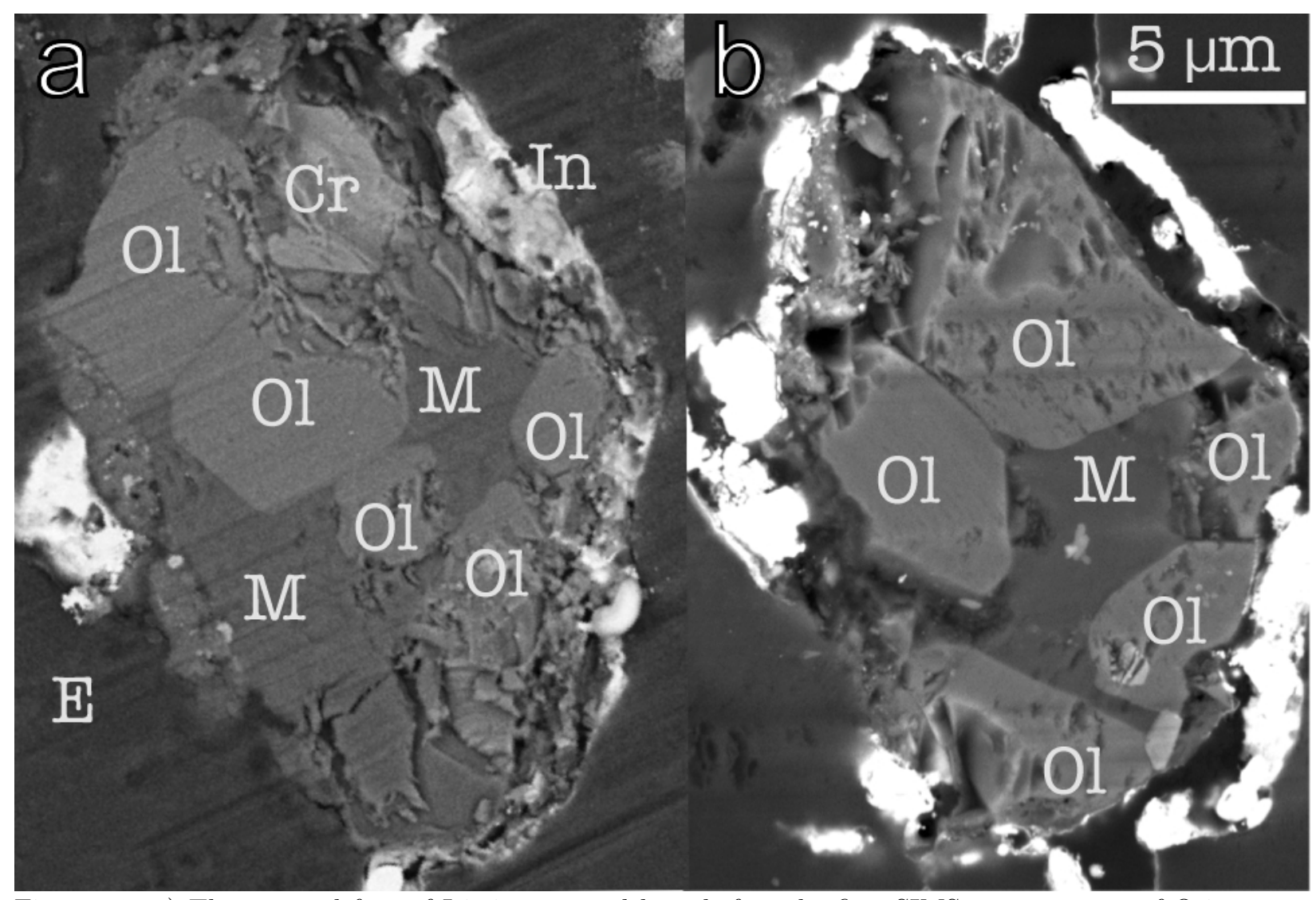

Figure 10: a) The exposed face of Iris in a potted butt before the first SIMS measurement of $\mathrm{O}$ isotopes. $\mathrm{Ol}=$ olivine, $\mathrm{Cr}=$ chromite, $\mathrm{M}=$ mesostasis, $\mathrm{In}=$ indium (embedding), $\mathrm{E}=$ epoxy (embedding). b) Iris after further microtoming, before the second SIMS measurement of $\mathrm{O}$ isotopes. 
( $2 \sigma$ uncertainties). Analyses of other olivines in Iris showed that they all have similar O composition. The second measurement of an olivine grain in the re-microtomed potted butt of Iris is $\delta^{17} \mathrm{O}=4.4 \pm 3.1 \%$ o, $\delta^{18} \mathrm{O}=6.3 \pm 2.4 \%$, consistent with the first measurement made 2.5 years prior (Figure 8). Uncertainties were slightly larger in our second measurement due to slightly worse reproducibility of the standard measurements.

\subsubsection{Bulb of Track 74: Reproducibility}

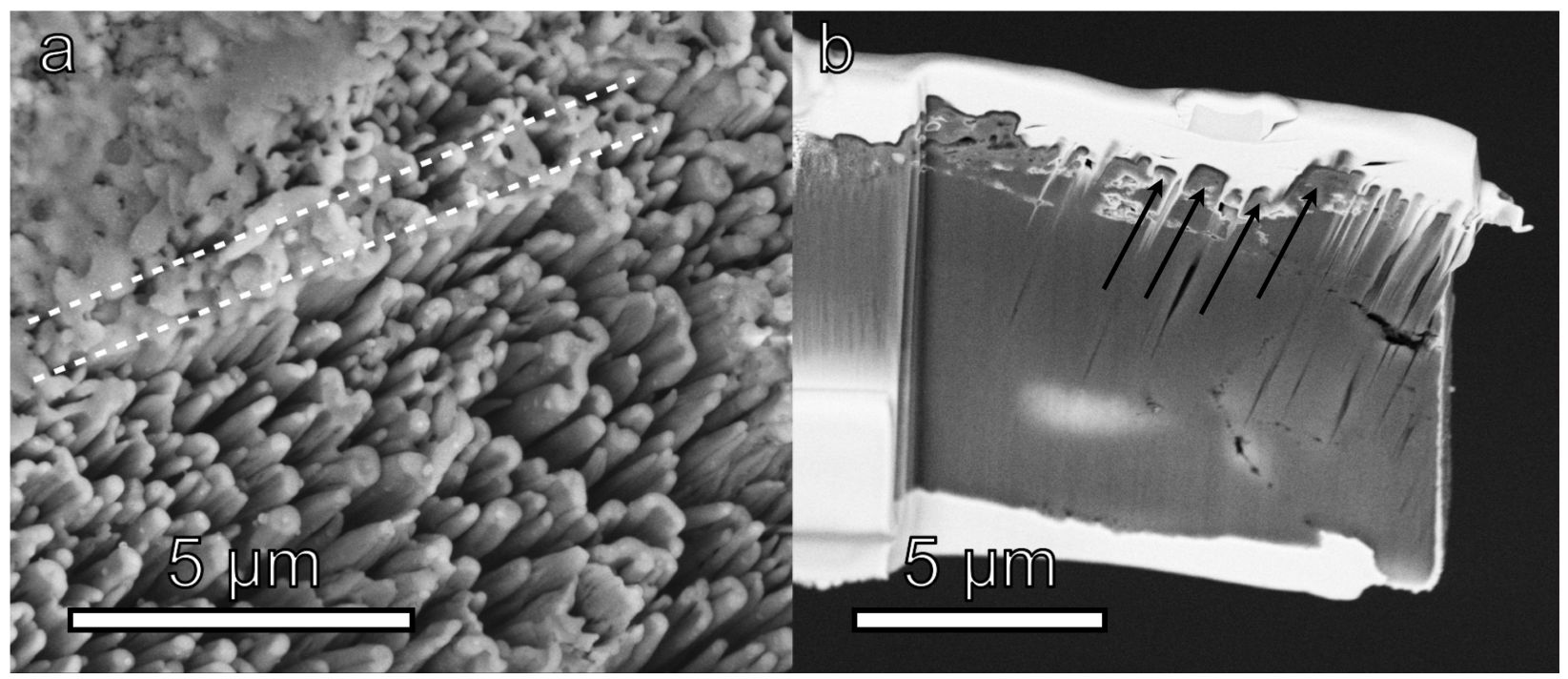

Figure 11: a) Secondary electron image of a region of compressed aerogel from the bulb of Stardust track C2052,74 after ion probe measurements. Dashed lines indicate the region of the FIB slice to remove the cometary material of interest. b) TEM bright-field image of the FIB slice after removal. White material at the top of the slice is deposited Pt, cometary particles are visible just below (four are indicated by black arrows).

Two Fe-rich $\sim 1-\mu \mathrm{m}$ particles in the wall of Stardust Track C2052,74 were measured to have high $\delta^{17,18} \mathrm{O}$ (and $\Delta^{17} \mathrm{O}$ ) compositions (Figure 9). We removed a slice of the compressed aerogel containing these particles and others by FIB, and placed the lamella on $\mathrm{Au}$ foil, exposing the particles for additional ion probe analysis (Figure 11). We identified Fe-rich particles (1-2 $\mu \mathrm{m}$ in size) in the FIB lamella by SEM-EDS. The chemical compositions of four particles were qualitatively consistent with the previous ion probe measurements. The particles were identified by high-angle annular dark field imaging in the TEM and were found to be amorphous by electron diffraction. It is possible that the particles were originally crystalline but were amorphized by the SIMS analysis.

We obtained isotope maps of three oxygen isotopes, ${ }^{16} \mathrm{OH}$, and ${ }^{56} \mathrm{Fe}^{16} \mathrm{O}$ of cometary material in this FIB slice, using a $<3 \mathrm{pA} \mathrm{Cs}^{+}$primary beam focused to $\sim 250 \mathrm{~nm}$, slightly lower beam 


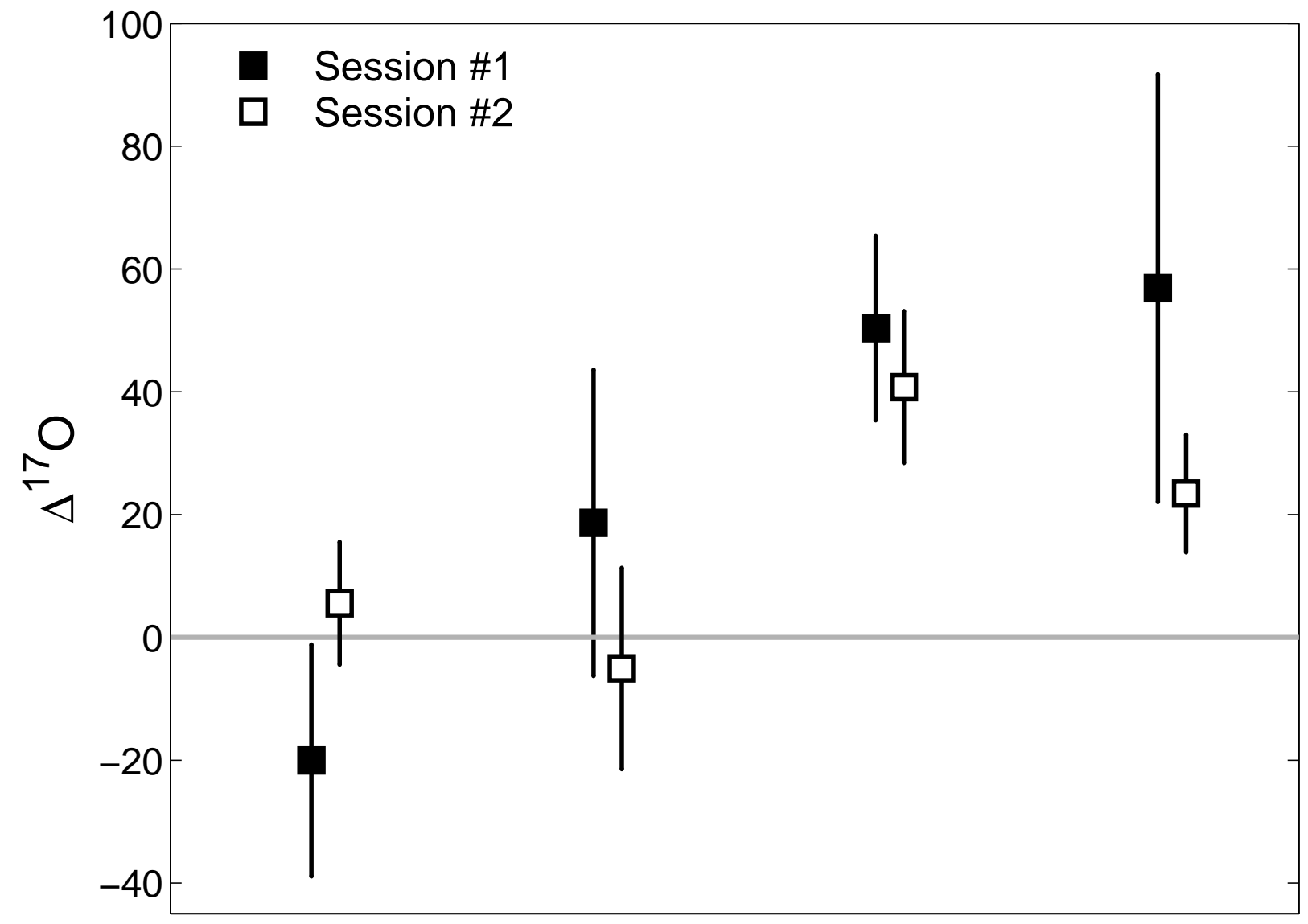

Figure 12: $\Delta^{17} \mathrm{O}$ of four $\mu \mathrm{m}$-sized particles from the wall of track $\mathrm{C} 2052,74$ made in compressed aerogel (filled squares: Session \#1) and in the same four particles in a removed FIB section (open squares: Session \#2). Uncertainties are $2 \sigma$. Session \#2 data are not included in Figure 9 and Table 5. 
current and spot size than used in the previous measurement. We compared the $\Delta^{17} \mathrm{O}$ of four particles in the FIB slice with measurements of those four particles in the aerogel. Mass-dependent instrumental fractionation due to the different sample mounting and analytical conditions will affect the measured $\delta^{17,18} \mathrm{O}$ of these four particles but should not affect $\Delta^{17} \mathrm{O}$. The FIB slice measurements generally confirmed the results of our first measurement of the four particles in aerogel, as shown in Figure 12. Discrepancies between these two measurements could be due to analyzing a different phase within a very small, multiphase particle, or effects from FIB sputtering or organometallic Pt deposition.

\section{Discussion}

\subsection{Coarse-grained material}

The spread of $\mathrm{O}$ isotopic compositions in the seven individual measured fragments is $\sim 19 \%$ in $\delta^{18} \mathrm{O}$ (excluding Tintin) with a standard deviation of $\sim 6 \%$. These measurements, made on fragments from five different tracks, reflect the diversity generally seen in Stardust samples in other analyses of, e.g., the chemical composition of Wild 2 olivine (Frank et al., 2014). Our O isotope measurements (again, excluding Tintin) are similar to the range of previous $\delta^{17,18} \mathrm{O}$ measurements of Wild 2 particles made by McKeegan et al. (2006); Nakamura et al. (2008); Nakashima et al. (2012a) (Figure 8). However, we did not measure any ${ }^{16} \mathrm{O}$-rich grains (e.g. $\delta^{17,18} \mathrm{O} \approx(-40,-40)$; McKeegan et al., 2006). These grains, often low-iron and manganese-enriched (LIME) olivines, appear to be much less common than ${ }^{16} \mathrm{O}$-poor Wild 2 grains, so this is not surprising.

Iris and Callie, two large terminal particles from Track C2052,74, are isotopically heavy, reflecting their likely origin as type-II chondrules (Ogliore et al., 2012; Gainsforth et al., in press).

Cecil has similar $\mathrm{O}$ isotopic composition as Callie and slightly lighter than Iris, but is from a different track. The igneous texture and mineralogy of portions of Cecil - Fe-rich olivines in a glassy matrix, with Fe-rich pyroxene and sulfides — shows some similarities to Iris and Callie. Further detailed study is required to know if some grains in Cecil are closely related to type II chondrule fragments, such as Iris and Callie, which appear to be common in comet Wild 2 (see also the Torajiro particle in Nakamura et al. (2008)).

Pooka, and The Butterfly are relatively simple pyroxene grains. Without the mineralogical context often seen in larger, multiphase Stardust terminal particles, it is difficult to know much 
about their origin. Both particles plot near the Young \& Russell line (Figure 8) with O isotopic compositions similar to Mg-rich chondrules (e.g. Tenner et al., 2015). Pooka and The Butterfly formed from a more ${ }^{16} \mathrm{O}$-rich reservoir than Cecil, Iris, and Callie. Ferromagnesian crystalline silicates from Wild 2 tend to show $\Delta^{17} \mathrm{O}$ values around $-2 \%$ for Fe-poor $(\mathrm{Mg} \#=94-97)$ fragments and slightly higher values $(\sim 0 \%)$ for more Fe-rich $(\mathrm{Mg} \#<85)$ fragments (Nakashima et al., 2012a). Only one of our particles had a $\mathrm{Mg} \#>85$ : The Butterfly, En 88 . This particle had the lowest $\Delta^{17} \mathrm{O}$ measured in the crystalline phases of the extracted fragments: $-3.4 \pm 2.2 \%$. Grains in Iris, Callie, and Pooka $\left(\mathrm{Mg} \#=64,64\right.$, and 80 , respectively) had higher $\Delta^{17} \mathrm{O}$ values consistent with $0 \%$. One $\mathrm{F}_{66}$ grain in Cecil plots just above the TFL $\left(\Delta^{17} \mathrm{O}=3.4 \pm 2.3 \%\right)$. The $\Delta^{17} \mathrm{O}$ of our five particles with crystalline ferromagnesian phases as a function of $\mathrm{Mg} /(\mathrm{Mg}+\mathrm{Fe})$ is plotted in Figure 13. Our measurements are consistent with the trend of $\Delta^{17} \mathrm{O}$ vs. $\mathrm{Mg} \#$ proposed by Nakashima et al. (2012a).

The amorphous silicate we measured in Caligula had O composition similar to Pooka and The Butterfly, indicating it formed from a similar O isotopic reservoir.

Tintin is amorphous and shows a curious vesiculated texture, which could be the result of flash heating during aerogel capture. It has a heterogenous $\mathrm{O}$ isotopic composition — one of the four measured spots was $13 \%$ greater in $\delta^{18} \mathrm{O}$ than the other three. We did not have an appropriate O isotope standard for the Fe-rich, Al-bearing silicate composition of Tintin. We used San Carlos olivine for the standard, which will result in a systematic uncertainty (due to matrix effects) of possibly up to a few \%o parallel to the TFL. Three of the four spots we measured were more than $10 \%$ isotopically light on the terrestrial fractionation line compared to the fourth spot.

The epoxy embedding medium has an isotopically light composition so contamination with the epoxy can potentially explain our Tintin measurements. Secondary electron images of Tintin after ion probe measurements showed that the measurement spots did not overlap with the surrounding epoxy, but in principle we could have measured epoxy that penetrated the interior of this vesiculated particle. However, we can rule this out using the mixing calculation given in Equation 4. Because Tintin's count rate $\left(\sim 2.5 \times 10^{6}\right)$ was much closer to a mineral standard $\left(\sim 3.5 \times 10^{6}\right.$ for San Carlos olivine) than the epoxy $\left(\sim 2.5 \times 10^{4}\right)$, the contamination of the measured $\mathrm{O}$ isotopic composition from epoxy has to be minimal: at most $\sim 1 \%$ in $\delta^{18} \mathrm{O}$ and $\sim 0.5 \%$ in $\delta^{17} \mathrm{O}$.

Tintin's variable and light measured $\mathrm{O}$ composition is likely the real composition of this particle, 


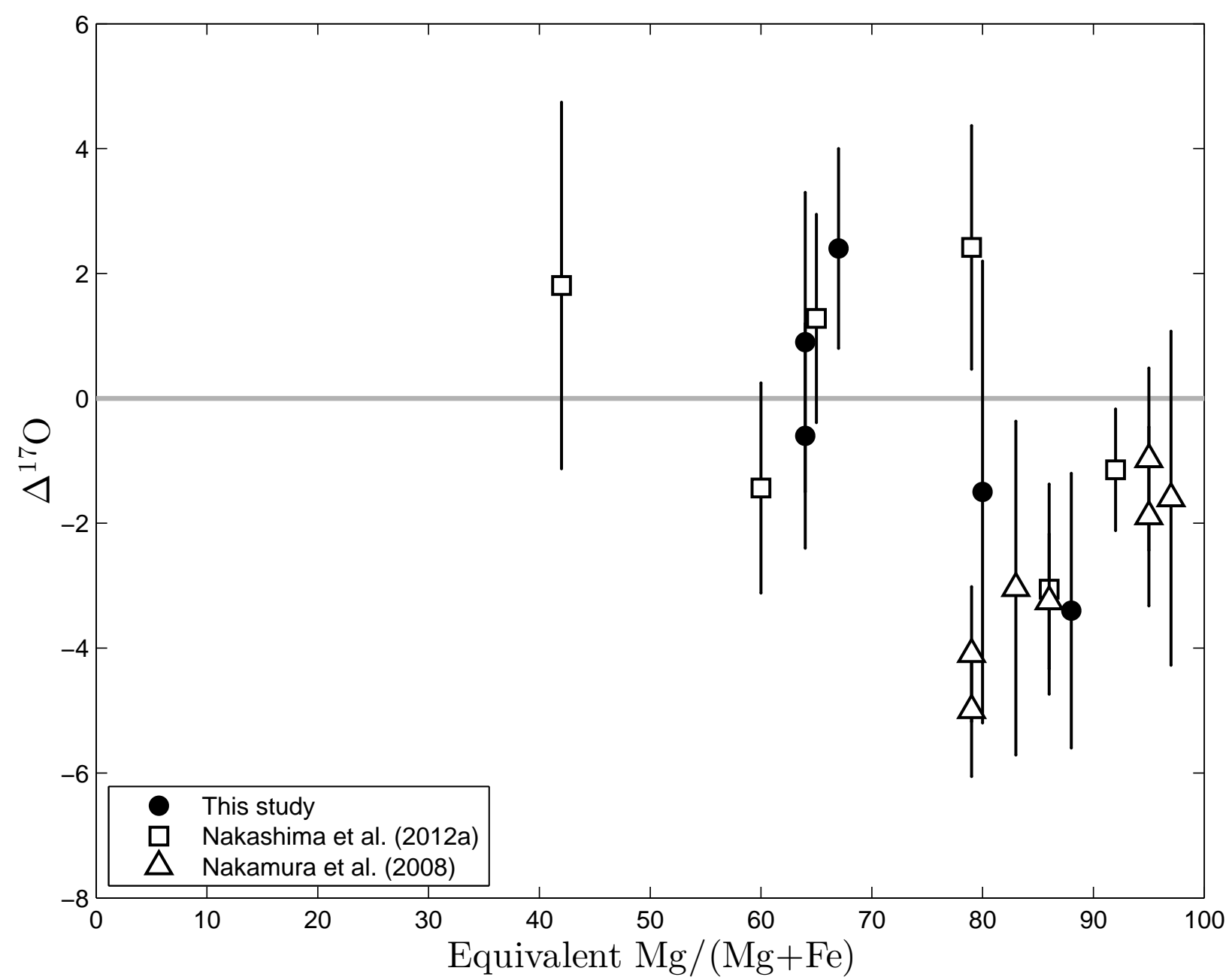

Figure 13: $\Delta^{17} \mathrm{O}$ vs. equivalent $\mathrm{Mg} /(\mathrm{Mg}+\mathrm{Fe})$ (or, $\left.\mathrm{Mg} \#\right)$ of Iris, Callie, Pooka, The Butterfly, and Cecil (filled circles) compared with literature data of other Wild 2 measurements, excluding ${ }^{16} \mathrm{O}$-rich particles (open squares: Nakashima et al. (2012a), open triangles: Nakamura et al. (2008)). Error bars are $2 \sigma$.

possibly altered from its pre-capture composition by heating/evaporative processes during aerogel capture.

\subsection{Presolar grain abundance}

We found no particles with extreme $\mathrm{O}$ or $\mathrm{C}$ isotopic anomalies clearly indicative of a circumstellar and presolar origin. While there are several small particles among the 63 measured in the bulb of track C2052,74 that deviate more than 50\% from the terrestrial O isotope ratios (Figure 9), the large majority of presolar grains have more-anomalous $\mathrm{O}$ isotopic composition: only $\sim 7 \%$ of presolar oxides and silicates in the presolar grain database (Hynes and Gyngard, 2009) were measured 
to be $-200 \%<<\delta^{18} \mathrm{O}<+200 \%$ and $-200 \% 0<\delta^{17} \mathrm{O}<+200 \%$. Here, we define presolar grains as $\left|\delta^{18} \mathrm{O}\right|>200 \%$ or $\left|\delta^{17} \mathrm{O}\right|>200 \%$. We identified 0 presolar grains in our measurements. If we assume the simplest case that the number of presolar grains in a sample of grains from comet Wild 2 are Poisson-distributed with mean $\lambda$, then the Poisson single-sided lower limit for the estimate of $\lambda$ is given by:

$$
\lambda \geq \frac{1}{2} \chi^{2}(\alpha ; 2 k)
$$

and similarly, the Poisson single-sided upper limit for the estimate of $\lambda$ is given by:

$$
\lambda \leq \frac{1}{2} \chi^{2}(1-\alpha ; 2 k+2)
$$

where $\chi^{2}$ is the inverse of the chi-square cumulative distribution function (we used chi2inv in Matlab 8.0.0.783) for confidence level $1-\alpha$ and observed number of events $k$. We found 0 presolar grains, so setting $k=0$, we calculate the single-sided upper limit at the $95 \%$ confidence level $(\alpha=0.05)$ to be 3.0 presolar grains, which equates to an abundance of $4.8 \%(3.0 / 63)$. (That is, the $95 \%$ confidence upper bound of the presolar grain abundance in Wild 2 fines, based on our measurements, is 4.8\%). One presolar grain, a silicon carbide, has been found in the Stardust aerogel (Brownlee et al., 2009). Clearly, many more measurements need to be made to ascertain a meaningful statistical upper bound of presolar grains in the comet Wild 2 aerogel fines.

The presolar grain abundance has been estimated from the Stardust foils, though care must be taken to account for presolar grain destruction caused by severe impact heating. Floss et al. (2013) estimated the presolar grain abundance in Stardust foil samples using high-speed analog shots of the Acfer 094 meteorite to account for presolar grain destruction. They found that the Acfer 094 impact residue was depleted in presolar grains compared to its nominal abundance and concluded that the same loss of presolar grains affected the Stardust foil samples. Floss et al. (2013) calculated the silicate+oxide presolar grain abundance in comet Wild 2 to be $600-830 \mathrm{ppm}$. This confidence interval represents only the range of Acfer 094 presolar grain abundance measurements, it does not take into account any statistical uncertainty. An estimate of the statistical uncertainty of these measurements, again assuming a Poisson distribution, yields a 95\% one-sided Poisson upper bound of $\sim 0.3 \%$, a much stricter upper bound than our measurements in the aerogel fines. Leitner et al. (2010) analyzed only small craters on the Stardust cometary foils (where dilution effects are small) 
and found one presolar grain, corresponding to a $2 \sigma$ (Poisson single-sided) upper limit of $0.8 \%$ on the presolar grain abundance.

At this point, extremely high abundances of isotopically anomalous presolar grains in comet Wild 2 cannot be ruled out. A few thousand ppm is possible in the bulk rocky material from the comet, and the fines can theoretically be much more enriched in presolar grains (percent-level) if the comet is a mixture of primitive fine-grained material and processed coarse-grained material.

\subsection{Fine-grained material}

The range of $\mathrm{O}$ compositions seen here in the bulb of Track 74 span the range of all known Solar-System materials, from very ${ }^{16} \mathrm{O}$-rich (mostly in Mg-rich, Fe-poor particles) to very ${ }^{16} \mathrm{O}-$ poor (in a high Fe region). Map \#3 contained particles which showed the largest variations in $\mathrm{O}$ isotopic composition. The ${ }^{16} \mathrm{O}$-poor, Fe-rich region in Map \#3 has $\mathrm{O}$ isotopic composition similar to cosmic symplectite $\left(\delta^{17,18} \mathrm{O}=+180 \%\right.$, Seto et al., 2008). However, cosmic symplectite is made of magnetite and pentlandite and the ${ }^{16} \mathrm{O}$-poor Wild 2 material is amorphous (Section 6.1.2). This Fe-rich material could have been an Fe-bearing crystalline object, such as magnetite, in the comet before being amorphized by high-speed capture or the ion probe measurements prior to TEM analysis. It is thought that Acfer 094 cosmic symplectite formed by the oxidation and sulfurization of Fe metal grains through interaction with outer nebular water (Seto et al., 2008). The anomalously ${ }^{17,18}$ O-rich Wild 2 material could have formed in a similar way before being incorporated into the comet.

Larger crystalline ferromagnesian silicates from comet Wild 2 show increasing $\Delta^{17} \mathrm{O}$ with increasing $\mathrm{Mg} /(\mathrm{Mg}+\mathrm{Fe}$ ) (Nakashima et al., 2012a), similar to $\mathrm{CR}$ chondrite chondrules. Track C2052,74 fines have highly variable $\Delta^{17} \mathrm{O}$ which does not correlate well with $\mathrm{Mg} /(\mathrm{Mg}+\mathrm{Fe})$ (correlation coefficient $=-0.14$ ) as shown in Figure 14, or with particle size (correlation coefficient $=$ 0.07) as shown in Figure 15. The error- and size-weighted bulk $\Delta^{17} \mathrm{O}$ of the Track C2052,74 fines is $4.5 \% \pm 2.9 \%(2 \sigma)$.

The fine-grained material in this track appears to sample a much broader range of $\mathrm{O}$ composition than seen in larger terminal particles. The standard deviation of $\delta^{18} \mathrm{O}$ of the 63 particles in our Stardust bulb measurements is $49 \%$, compared to the $\sim 7 \%$ standard deviation of our coarsegrained measurements (albeit from a much smaller sample size). From our isotopic measurements and mineralogical studies of bulb fines by Stodolna et al. (2012), we conclude that the fine-grained 


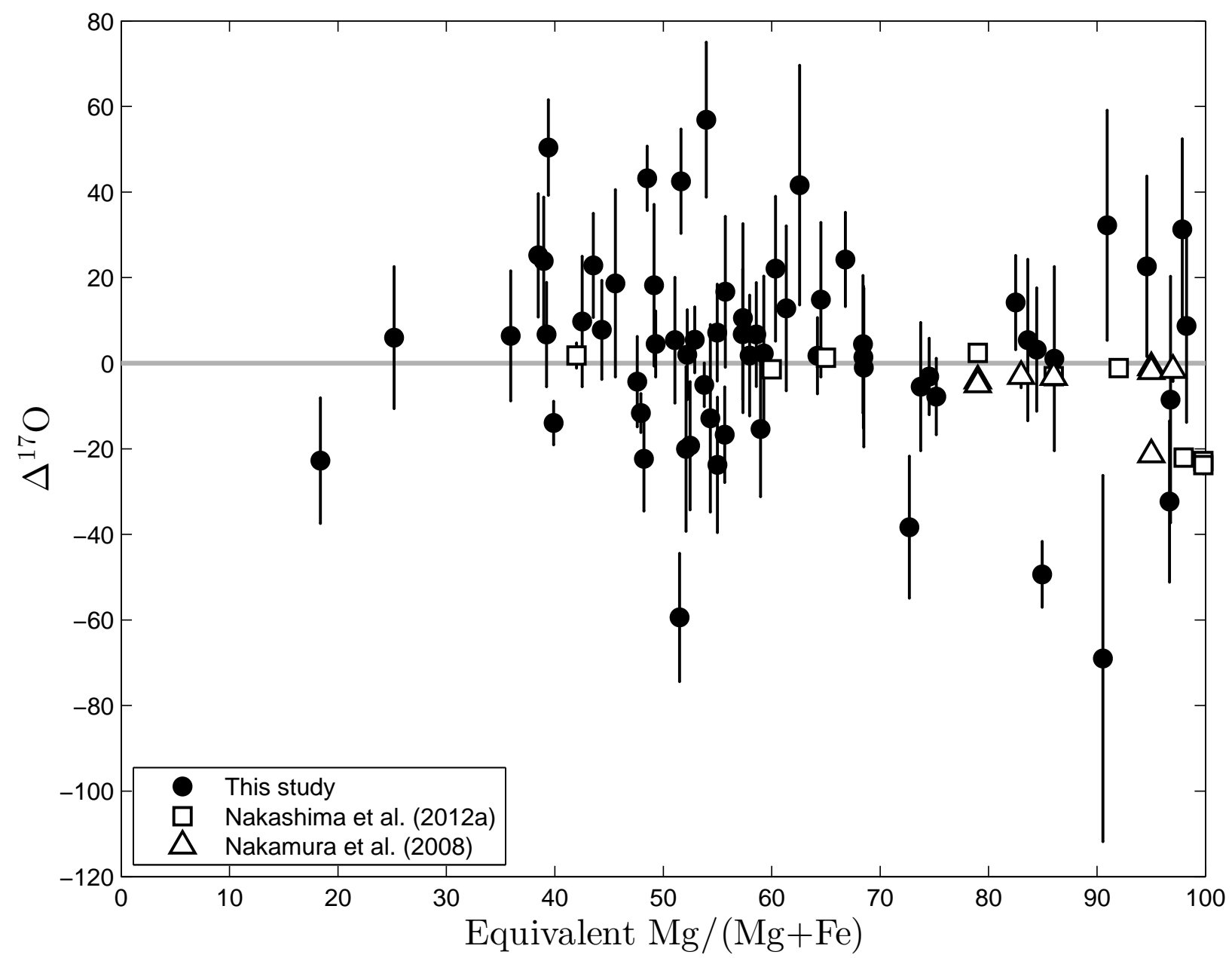

Figure 14: $\Delta^{17} \mathrm{O}$ vs. equivalent $\mathrm{Mg} /(\mathrm{Mg}+\mathrm{Fe}$ ) (or, $\mathrm{Mg} \#)$ of 63 particles from the bulb of Stardust Track C2052,74 (filled circles: this study) compared with literature data (open squares: Nakashima et al. (2012a), open triangles: Nakamura et al. (2008)). Error bars are $2 \sigma$.

Wild 2 material is not simply a smaller-size representation of the larger Wild 2 grains.

Nakashima et al. (2012b) measured the O isotopic composition of an anhydrous interplanetary dust particle (Z17) that showed distinct $\Delta^{17} \mathrm{O}$ between its fine-grained $(-4.3 \pm 0.9,2 \sigma)$ and coarse-grained $(-0.4 \pm 1.4)$ components. The fine component of anhydrous interplanetary dust may have sampled different $\mathrm{O}$ isotopic reservoirs than the larger mineral grains, analogous to our measurements of the fine- and coarse-grained components of comet Wild 2.

To understand the provenance of the fine-grained material from comet Wild 2, it is also useful to look at our measurements in the context of asteroidal fines. If fine-grained material in the early Solar System was ubiquitous at all radii, we would expect the Wild 2 fines to be similar to the 


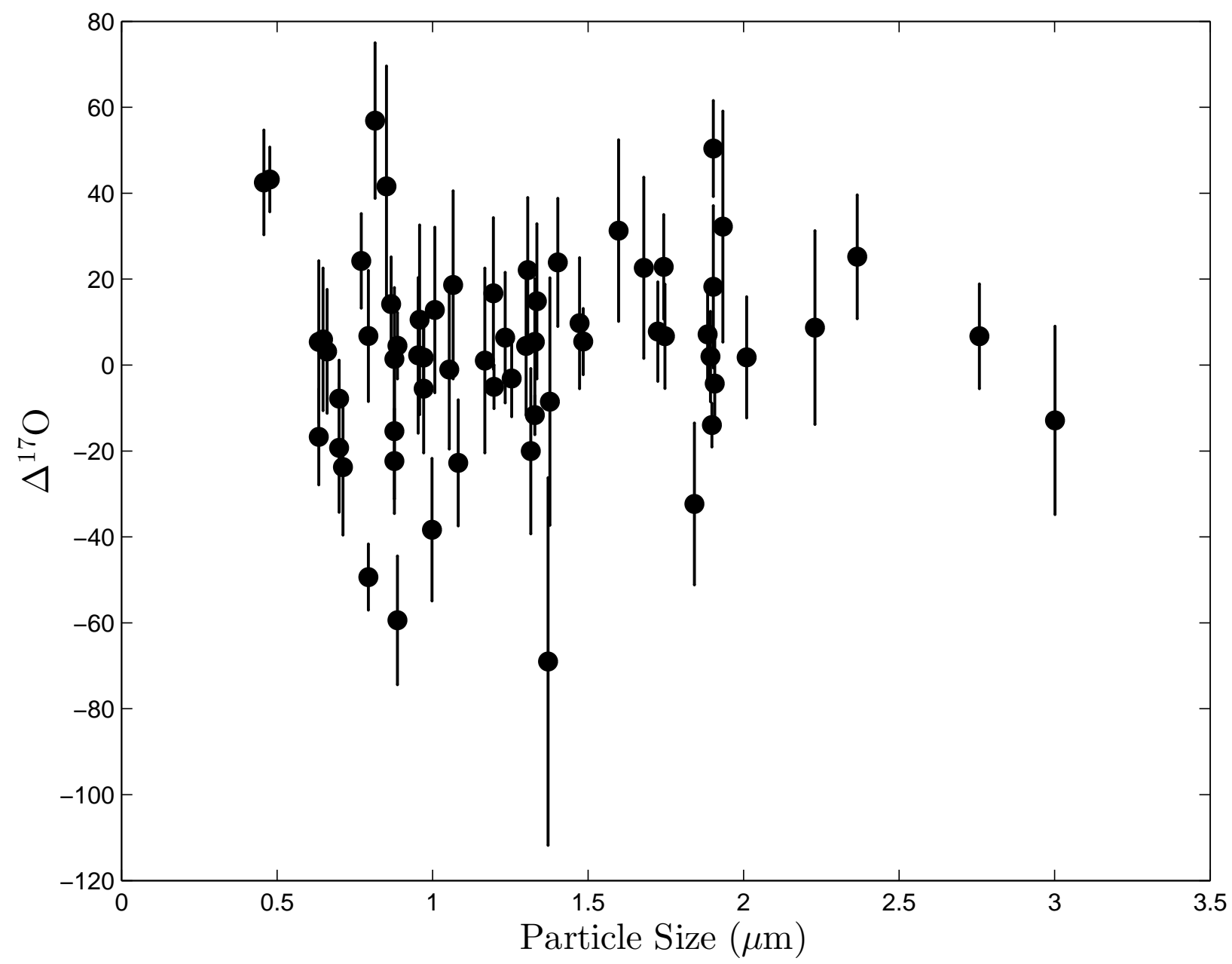

Figure 15: $\Delta^{17} \mathrm{O}$ vs. particle size of 63 particles from the bulb of Stardust Track C2052,74. Error bars are $2 \sigma$.

matrix of primitive meteorites. If, instead, the Wild 2 fines sampled a different reservoir of $\mathrm{O}$ than asteroids did, we would expect to see differences in their $\mathrm{O}$ composition. In particular, is the diversity of $\mathrm{O}$ compositions sampled by the Wild 2 fines seen in the fines in another cometary or asteroidal parent body? Interplanetary dust particles are samples of a large number of parent bodies, not a single asteroid or comet, so in the following discussion we will compare our Wild 2 measurements with fine-grained material in meteorites.

Kunihiro et al. (2005) measured the O isotopic composition of the Vigarano meteorite, a CV3 chondrite that has undergone mild thermal and aqueous alteration (Lee et al., 1996). They determined that the upper bound standard deviation (limited by the measurement's statistical error) of the very fine-grained ( $<500 \mathrm{~nm}$ grains) groundmass matrix of Vigarano is $5 \%$ in $\delta^{18} \mathrm{O}$. This 
is much smaller than the $49 \%$ standard deviation from our measurements, indicating that the fine-grained material in comet Wild 2 likely sampled a more diverse source of $\mathrm{O}$ isotopes than the fine-grained material in the CV parent asteroid. However, the fines in CV3 meteorites likely experienced weak thermal and aqueous processing on the asteroid which may have partially equilibrated $\mathrm{O}$ isotopes and produced a relatively narrow distribution of $\mathrm{O}$ isotopic compositions. There was likely no significant thermal or aqueous processing on comet Wild 2 which would produce a narrow, Vigarano-like distribution of $\mathrm{O}$ isotopes in the Wild 2 fines. A study of the $\mathrm{O}$ isotope composition of individual matrix grains of primitive meteorites that experienced minimal alteration, such as the CR chondrites Queen Alexandra Range (QUE) 99177 and Meteorite Hills (MET) 00426 (Abreu and Brearley, 2010) or the ungrouped carbonaceous chondrite Acfer 094 (Greshake, 1997), would make for a more appropriate comparison with the comet Wild 2 fines.

Presolar grains and small refractory matrix grains in primitive meteorites escaped homogenization during chondrule formation. Takigawa et al. (2014) measured the O isotopic composition of 107 alumina grains ( $\sim 95 \%$ were corundum) $1 \mu \mathrm{m}$ and smaller from unequilibrated ordinary chondrites. They found a very large spread in O composition for the solar corundum grains (Figure 9 of Takigawa et al. (2014)), including very ${ }^{16} \mathrm{O}$-rich and ${ }^{16} \mathrm{O}$-poor grains. The standard deviation of $\delta^{18} \mathrm{O}$ for these grains is $\sim 32 \%$. These measurements of corundum grains in UOCs are similar to the spread of $\mathrm{O}$ compositions we measured in the fine-grained component of comet Wild 2.

Makide et al. (2009b) measured 109 larger $(1-5 \mu \mathrm{m})$ corundum grains and found them to have ${ }^{16} \mathrm{O}$-rich compositions (mean $\Delta^{17} \mathrm{O}=-22 \%$ ) with $15 \%$ standard deviation in $\delta^{18} \mathrm{O}$. These corundum grains appear to be more homogenous and ${ }^{16} \mathrm{O}$-rich than the smaller corundum grains measured by Takigawa et al. (2014). At larger size scales, refractory inclusions like AOAs and CAIs also have relatively uniform ${ }^{16} \mathrm{O}$-rich compositions. The larger objects, CAIs, AOAs, and larger corundum grains, likely formed in the solar nebula close to the young Sun. (Some corundum grains from carbonaceous chondrites formed via hydrothermal alteration on a parent body and have O isotopic compositions consistent with the terrestrial fractionation line (e.g. Maruyama et al., 2008)). The small corundum grains measured by Takigawa et al. (2014) may be circumstellar grains, but lack the nucleosynthetic isotope anomalies that are typically used to label them as presolar. (The isotopically anomalous grains measured by Takigawa et al. (2014) mostly had rough surface morphology possibly caused by energetic particle irradiation in interstellar space. The 
corundum grains with rough surfaces but without extreme isotope anomalies may have had similar origins and histories as the anomalous grains.) These corundum grains could be a component of the solids inherited from our parent molecular cloud, reflecting the diverse $\mathrm{O}$ isotopic compositions of the primitive constituents of the Solar System. Corundum grains survived processing on the UOC parent body and were able to retain their original, diverse $\mathrm{O}$ isotopic composition because self-diffusion of O in corundum is very slow (Heuer, 2008). Other small, less robust molecular cloud grains were destroyed or their O isotopic signatures were erased by nebular and/or asteroidal processes and are not found in meteorites.

Our measurements of a very wide range of $\mathrm{O}$ isotopic compositions in the Wild 2 fine-grained material, covering the entire range of known Solar-System materials, can be explained by one or a combination of the following two scenarios: 1) The Wild 2 fines sampled many oxygen reservoirs from the inner solar nebula, or 2) the fines are representative of the raw starting materials, inherited from the parent molecular cloud, from which the inner Solar System material formed.

\subsubsection{Scenario \#1: Comet Wild 2 fines sampled many oxygen reservoirs from the inner} Solar System

In this scenario, the comet Wild 2 fines formed in the inner solar nebula and were transported to the outer nebula where they were incorporated into the comet. The fine-grained material in the outer Solar System at the time of the comet's formation was mostly of foreign origin, implying that the transport of inner Solar System material to the outer solar nebula was efficient. Other fine-grained material that was present in the time and place where the comet formed, i.e. surviving molecular cloud solids, would have been incorporated into the comet as well. But if most of the comet Wild 2 fines are of foreign provenance, the amount of surviving molecular cloud material would be small compared to the inner Solar System material.

We can make testable predictions if the Wild 2 fines are from the inner Solar System. Presolar grains are more likely to survive in the cold outer regions of the solar nebula compared to the inner nebula where they are destroyed by nebular processing. Therefore, the presolar grain abundances of inner Solar System material should be less than unprocessed outer nebula material. In this scenario, the presolar grain abundances in Wild 2 fines should be comparable to the abundances in primitive meteorites, which incorporated inner Solar System material but did not destroy presolar grains through heating or aqueous alteration on the parent asteroid. However, as described in 
Section 7.2 , the statistical uncertainty of the presolar grain abundance in a given parent body can be very large due to limited sampling.

Volatile species are more easily retained in the cold outer nebula compared in the inner nebula. Most meteorites are depleted in volatile elements relative to the Sun, except for CI chondrites which escaped high-temperature processing and have chemical composition similar to the solar photosphere. The volatile depletion patterns seen in the various chondrite classes were likely caused by variable heating of material in the protoplanetary accretion disk (Davis, 2006). If Wild 2 fines were sourced from the inner Solar System, they would also be depleted in volatiles compared to CI chondrites.

7.3.2. Scenario \#2: Comet Wild 2 fines are representative of the raw starting materials, inherited from the parent molecular cloud, from which the inner Solar System material formed

Conversely, if the fines in Wild 2 were not transported from the inner Solar System, we would draw different conclusions about the nature of material in the solar nebula at large distances from the Sun, and make different predictions about the presolar grain abundances and volatile depletion in Wild 2 fines. In this scenario, the fine component would be dominated by grains inherited from the Solar System's parent molecular cloud (e.g. circumstellar grains with nucleosynthetic signatures, amorphous interstellar silicates). Abudances of isotopically anomalous presolar grains would be high compared to primitive meteorites and volatiles would not be significantly depleted compared to CI chondrites.

Our current measurements do not strongly support Scenario \#1 or \#2. Presolar grain abundances and the bulk chemical composition of the Wild 2 fines are currently too imprecise to compare to measurements of primitive meteorites.

The diverse $\mathrm{O}$ isotopic compositions of the fines were not equilibrated on the comet because it was kept in cold storage beyond the orbit of Neptune since the Solar System's formation. Our measurements of diverse $\mathrm{O}$ isotopic compositions agree with TEM studies of Wild 2 fines by Stodolna et al. (2012) which found unequilibrated, heterogenous particles with approximately chondritic average chemical composition.

The larger terminal particles collected by Stardust have high-temperature mineralogy and ${ }^{16} \mathrm{O}-$ poor compositions that indicate they formed in the inner Solar System and were transported to 
the scattered disk where they were incorporated into comet Wild 2 . The lack of ${ }^{26} \mathrm{Al}$ in four Wild 2 fragments measured so far (Matzel et al., 2010; Ishii et al., 2010; Ogliore et al., 2012; Nakashima et al., 2015) tells us that this transport happened relatively late, more than 3 Myr after the onset of CAI formation. These coarse-grained inner Solar System objects combined with primitive dust and ices in the cold, sparse, scattered disk, and eventually accreted together to form comet Wild 2.

It should be noted that our measurements of the 63 bulb particles from comet Wild 2 represent only a small section of the bulb of only one of hundreds of tracks returned by the Stardust mission. Similarly, there have only been $\sim 25$ larger Wild 2 particles measured for O isotopes (McKeegan et al., 2006; Nakamura et al., 2008; Messenger et al., 2008; Nakamura-Messenger et al., 2011; Nakashima et al., 2012a). The diversity seen in these cometary samples so far underscores the need for more $\mathrm{O}$ isotope and chemical analyses of both the terminal particles and the fines. There have been many more analyses of a given individual type of chondrite, which represents a much more homogeneous parent body than comet Wild 2. Many more tracks need to be analyzed before we can confidently understand the composition of this comet.

\section{Conclusions}

We measured the $\mathrm{O}$ isotopic of composition seven $>2 \mu \mathrm{m}$ fragments extracted from five Stardust aerogel tracks, and 63 small particles $(<2 \mu \mathrm{m})$ measured in compressed aerogel from the bulb of track C2052,74. The larger fragments have O isotopic compositions mostly consistent with the terrestrial fractionation line. The fine-grained material shows a very diverse $\mathrm{O}$ isotopic composition that spans the range of all Solar System materials, and is similar to the O isotope distribution seen in $<1 \mu \mathrm{m}$ corundum grains extracted from unequilibrated ordinary chondrites. We conclude that the larger particles were formed in the inner Solar System from an evolved O isotopic reservoir, and transported to the scattered disk. These larger particles then combined with unequilibrated dust of diverse O isotopic composition, inherited from the Solar System's parent molecular cloud or from a diverse sample of inner Solar System oxygen reservoirs, to form the rocky component of comet Wild 2 . 


\section{Acknowledgements}

The authors thank Daisuke Nakashima, Larry Nittler, and an anonymous reviewer for their suggestions that helped improved this paper. The National Center for Electron Microscopy is supported by the Director, Office of Science, Office of Basic Energy Sciences, of the U.S. Department of Energy, under Contract No. DE-AC02-05CH11231. This work was supported by NASA grants NNX07AM62G (G.R.H.), NNX07AM67G (A.J.W.), and NNX14AF24G (R.C.O.). 


\section{References}

Abreu, N.M., Brearley, A.J., 2010. Early solar system processes recorded in the matrices of two highly pristine CR3 carbonaceous chondrites, MET 00426 and QUE 99177. Geochimica et Cosmochimica Acta 74, 1146-1171.

Bottazzi, P., Ottolini, L., Vannucci, R., 1992. SIMS analyses of rare earth elements in natural minerals and glasses: An investigation of structural matrix effects on ion yields. Scanning 14, 160-168.

Brownlee, D., 2014. The Stardust Mission: Analyzing samples from the edge of the solar system. Annual Review of Earth and Planetary Sciences 42, 179-205.

Brownlee, D., Joswiak, D., Matrajt, G., 2012. Overview of the rocky component of Wild 2 comet samples: Insight into the early solar system, relationship with meteoritic materials and the differences between comets and asteroids. Meteoritics \& Planetary Science 47, 453-470.

Brownlee, D., Joswiak, D., Matrajt, G., Messenger, S., Ito, M., 2009. Silicon carbide in comet Wild $2 \&$ the abundance of pre-solar grains in the Kuiper belt, in: Lunar and Planetary Science Conference, p. 2195.

Brownlee, D., Tsou, P., Aléon, J., Alexander, C.M., Araki, T., Bajt, S., Baratta, G.A., Bastien, R., Bland, P., Bleuet, P., et al., 2006. Comet 81P/Wild 2 under a microscope. Science 314, 1711-1716.

Burchell, M., Fairey, S., Wozniakiewicz, P., Brownlee, D., Hörz, F., Kearsley, A., See, T., Tsou, P., Westphal, A., Green, S., et al., 2008. Characteristics of cometary dust tracks in Stardust aerogel and laboratory calibrations. Meteoritics \& Planetary Science 43, 23-40.

Ciesla, F.J., 2007. Outward transport of high-temperature materials around the midplane of the solar nebula. science $318,613-615$.

Clayton, R.N., 2002. Solar system: Self-shielding in the solar nebula. Nature 415, 860-861.

Clayton, R.N., Onuma, N., Grossman, L., Mayeda, T.K., 1977. Distribution of the pre-solar component in Allende and other carbonaceous chondrites. Earth and Planetary Science Letters 34, 209-224. 
Davis, A.M., 2006. Volatile evolution and loss. Meteorites and the Early Solar System II 1, 295-307.

Floss, C., Stadermann, F.J., Kearsley, A.T., Burchell, M.J., Ong, W.J., 2013. The abundance of presolar grains in comet 81P/Wild 2. The Astrophysical Journal 763, 140.

Frank, D.R., Zolensky, M.E., Le, L., 2014. Olivine in terminal particles of Stardust aerogel tracks and analogous grains in chondrite matrix. Geochimica et Cosmochimica Acta 142, 240-259.

Gainsforth, Z., Butterworth, A., Stodolna, J., Westphal, A., Huss, G., Nagashima, K., Ogliore, R., Brownlee, D., Joswiak, D., Tylczszak, T., Simionovici, A., in press. Constraints on the formation environment of two chondrule-like igneous particles from Comet 81P/Wild 2. Meteoritics and Planetary Science .

Gainsforth, Z., McLeod, A., Butterworth, A., Dominguez, G., Basov, D., Keilmann, F., Thiemens, M., Tyliszczak, T., Westphal, A., 2013. Caligula, a Stardust sulfide-silicate assemblage viewed through SEM, nanoFTIR, and STXM, in: Lunar and Planetary Institute Science Conference Abstracts, p. 2332.

Gainsforth, Z., Ogliore, R., Bustillo, K., Westphal, A., Butterworth, A., 2014. Ni zoned nanopyrrhotite from Stardust track C2062,2,162 (Cecil), in: Lunar and Planetary Institute Science Conference Abstracts, p. 2637.

Greshake, A., 1997. The primitive matrix components of the unique carbonaceous chondrite Acfer 094: A TEM study. Geochimica et Cosmochimica Acta 61, 437-452.

Heuer, A., 2008. Oxygen and aluminum diffusion in $\alpha-\mathrm{Al}_{2} \mathrm{O}_{3}$ : How much do we really understand? Journal of the European Ceramic Society 28, 1495-1507.

Huss, G., 2012. On the differences in isotopic compositions of O and N between the Sun and inner solar system solids revealed by Genesis. Meteoritics and Planetary Science Supplement 75, 5294 .

Hynes, K., Gyngard, F., 2009. The presolar grain database: http://presolar.wustl.edu/ pgd, in: Lunar and Planetary Science Conference, p. 1198.

Ishii, H., Stadermann, F., Floss, C., Joswiak, D., Bradley, J., Teslich, N., Brownlee, D., Matrajt, G., MacPherson, G., McKeegan, K., 2010. Lack of evidence for in situ decay of Aluminum-26 
in comet $81 \mathrm{P} /$ Wild 2 CAI-like refractory particles 'Inti' and 'Coki', in: Lunar and Planetary Institute Science Conference Abstracts, p. 2317.

Jogo, K., Nagashima, K., Hutcheon, I.D., Krot, A.N., Nakamura, T., 2012. Heavily metamorphosed clasts from the CV chondrite breccias Mokoia and Yamato-86009. Meteoritics \& Planetary Science 47, 2251-2268.

Jones, R.H., 1990. Petrology and mineralogy of type II, FeO-rich chondrules in Semarkona (LL3. 0): Origin by closed-system fractional crystallization, with evidence for supercooling. Geochimica et Cosmochimica Acta 54, 1785-1802.

Joswiak, D.J., Brownlee, D.E., Matrajt, G., Westphal, A.J., Snead, C.J., Gainsforth, Z., 2012. Comprehensive examination of large mineral and rock fragments in Stardust tracks: Mineralogy, analogous extraterrestrial materials, and source regions. Meteoritics \& Planetary Science 47, $471-524$.

Kita, N.T., Ushikubo, T., Fu, B., Valley, J.W., 2009. High precision sims oxygen isotope analysis and the effect of sample topography. Chemical Geology 264, 43-57.

Kunihiro, T., Nagashima, K., Yurimoto, H., 2005. Microscopic oxygen isotopic homogeneity/heterogeneity in the matrix of the Vigarano CV3 chondrite. Geochimica et Cosmochimica Acta $69,763-773$.

Lee, M., Hutchison, R., Graham, A., 1996. Aqueous alteration in the matrix of the vigarano (CV3) carbonaceous chondrite. Meteoritics \& Planetary Science 31, 477-483.

Leitner, J., Hoppe, P., Heck, P., 2010. First discovery of presolar material of possible supernova origin in impact residues from comet 81P/Wild 2. 41st LPSC , \#1607.

Makide, K., Nagashima, K., Krot, A., Huss, G., 2009a. Oxygen isotopic compositions of solar, micrometer-sized corundum, hibonite and spinel grains in acid-resistant residues from ordinary and carbonaceous chondrites. 40th LPSC , \#2079.

Makide, K., Nagashima, K., Krot, A.N., Huss, G.R., 2009b. Oxygen isotopic compositions of solar corundum grains. The Astrophysical Journal 706, 142. 
Maruyama, S., Kunihiro, T., Nakamura, E., 2008. A hercynite-rich inclusion in the Vigarano CV3 chondrite. Meteoritics and Planetary Science Supplement 43, 5183.

Matzel, J., Ishii, H., Joswiak, D., Hutcheon, I., Bradley, J., Brownlee, D., Weber, P., Teslich, N., Matrajt, G., McKeegan, K., et al., 2010. Constraints on the formation age of cometary material from the NASA Stardust mission. Science 328, 483-486.

McKeegan, K., Aléon, J., Bradley, J., Brownlee, D., Busemann, H., Butterworth, A., Chaussidon, M., Fallon, S., Floss, C., Gilmour, J., 2006. Isotopic compositions of cometary matter returned by Stardust. Science 314, 1724-1728.

McKeegan, K., Kallio, A., Heber, V., Jarzebinski, G., Mao, P., Coath, C., Kunihiro, T., Wiens, R., Nordholt, J., Moses, R., et al., 2011. The oxygen isotopic composition of the sun inferred from captured solar wind. Science 332, 1528-1532.

Messenger, S., Ito, M., Joswiak, D.J., Keller, L.P., Stroud, R.M., Nakamura-Messenger, K., Matrajt, G., Brownlee, D.E., 2008. Oxygen Isotopic Compositions of Wild 2 Silicates. Meteoritics and Planetary Science Supplement 43, 5308.

Nakamura, T., Noguchi, T., Tsuchiyama, A., Ushikubo, T., Kita, N.T., Valley, J.W., Zolensky, M.E., Kakazu, Y., Sakamoto, K., Mashio, E., Uesugi, K., Nakano, T., 2008. Chondrulelike objects in short-period comet 81P/Wild 2. Science 321, 1664-1667.

Nakamura-Messenger, K., Keller, L.P., Clemett, S.J., Messenger, S., Ito, M., 2011. Nanometer-scale anatomy of entire Stardust tracks. Meteoritics \& Planetary Science 46, 1033-1051.

Nakashima, D., Ushikubo, T., Joswiak, D.J., Brownlee, D.E., Matrajt, G., Weisberg, M.K., Zolensky, M.E., Kita, N.T., 2012a. Oxygen isotopes in crystalline silicates of comet Wild 2: A comparison of oxygen isotope systematics between Wild 2 particles and chondritic materials. Earth and Planetary Science Letters 357, 355-365.

Nakashima, D., Ushikubo, T., Kita, N.T., Weisberg, M.K., Zolensky, M.E., Ebel, D.S., 2015. Late formation of a comet Wild 2 crystalline silicate particle, Pyxie, inferred from $\mathrm{Al}-\mathrm{Mg}$ chronology of plagioclase. Earth and Planetary Science Letters 410, 54-61. 
Nakashima, D., Ushikubo, T., Zolensky, M.E., Kita, N.T., 2012b. High precision oxygen threeisotope analyses of anhydrous chondritic interplanetary dust particles. Meteoritics \& Planetary Science 47, 197-208.

Ogliore, R.C., Huss, G., Nagashima, K., Butterworth, A., Gainsforth, Z., Stodolna, J., Westphal, A., Joswiak, D., Tyliszczak, T., 2012. Incorporation of a late-forming chondrule into comet wild 2. The Astrophysical Journal Letters 745, L19.

Seto, Y., Sakamoto, N., Fujino, K., Kaito, T., Oikawa, T., Yurimoto, H., 2008. Mineralogical characterization of a unique material having heavy oxygen isotope anomaly in matrix of the primitive carbonaceous chondrite Acfer 094. Geochimica et Cosmochimica Acta 72, 2723-2734.

Simon, S.B., Joswiak, D.J., Ishii, H.A., Bradley, J.P., Chi, M., Grossman, L., Aléon, J., Brownlee, D.E., Fallon, S., Hutcheon, I.D., Matrajt, G., McKeegan, K.D., 2008. A refractory inclusion returned by Stardust from comet 81P/Wild 2. Meteoritics \& Planetary Science 43, 1861-1877.

Stodolna, J., Gainsforth, Z., Leroux, H., Butterworth, A.L., Tyliszczak, T., Jacob, D., Westphal, A.J., 2013. Iron valence state of fine-grained material from the Jupiter family comet 81P/Wild 2-a coordinated TEM/STEM EDS/STXM study. Geochimica et Cosmochimica Acta 122, 1-16.

Stodolna, J., Jacob, D., Leroux, H., 2012. Mineralogy and petrology of Stardust particles encased in the bulb of track 80: TEM investigation of the Wild 2 fine-grained material. Geochimica et Cosmochimica Acta 87, 35-50.

Takigawa, A., Tachibana, S., Huss, G.R., Nagashima, K., Makide, K., Krot, A.N., Nagahara, H., 2014. Morphology and crystal structures of solar and presolar $\mathrm{Al}_{2} \mathrm{O}_{3}$ in unequilibrated ordinary chondrites. Geochimica et Cosmochimica Acta 124, 309-327.

Tenner, T.J., Nakashima, D., Ushikubo, T., Kita, N.T., Weisberg, M.K., 2015. Oxygen isotope ratios of FeO-poor chondrules in cr3 chondrites: Influence of dust enrichment and $\mathrm{H}_{2} \mathrm{O}$ during chondrule formation. Geochimica et Cosmochimica Acta 148, 228-250.

Westphal, A.J., Butterworth, A., Gainsforth, Z., Huss, G.R., Nagashima, K., Ogliore, R.C., Stodolna, J., 2011. Reversible sample preparation of small particles in potted butts for sims analyses. 74th Annual Meeting of the Meteoritical Society 74, 5274 . 
Westphal, A.J., Fakra, S.C., Gainsforth, Z., Marcus, M.A., Ogliore, R.C., Butterworth, A.L., 2009. Mixing fraction of inner solar system material in comet 81P/Wild2. The Astrophysical Journal $694,18-28$.

Westphal, A.J., Gainsforth, Z., Goldstein, J.I., Humayun, M., Kunz, M., Marcus, M.A., Stroud, R.M., Tamura, N., 2012. Simeio: An Ultra Ni-Poor Metal Particle from Comet 81P/Wild2. Meteoritics and Planetary Science Supplement 75, 5309.

Westphal, A.J., Snead, C., Butterworth, A., Graham, G.A., Bradley, J.P., Bajt, S., Grant, P.G., Bench, G., Brennan, S., Pianetta, P., 2004. Aerogel keystones: Extraction of complete hypervelocity impact events from aerogel collectors. Meteoritics \& Planetary Science 39, 1375-1386.

Young, E.D., Russell, S.S., 1998. Oxygen reservoirs in the early solar nebula inferred from an Allende CAI. Science 282, 452-455.

Zolensky, M.E., Zega, T.J., Yano, H., Wirick, S., Westphal, A.J., Weisberg, M.K., Weber, I., Warren, J.L., Velbel, M.A., Tsuchiyama, A., Tsou, P., Toppani, A., Tomioka, N., Tomeoka, K., Teslich, N., Taheri, M., Susini, J., Stroud, R., Stephan, T., Stadermann, F.J., Snead, C.J., Simon, S.B., Simionovici, A., See, T.H., Robert, F., Rietmeijer, F.J.M., Rao, W., Perronnet, M.C., Papanastassiou, D.A., Okudaira, K., Ohsumi, K., Ohnishi, I., Nakamura-Messenger, K., Nakamura, T., Mostefaoui, S., Mikouchi, T., Meibom, A., Matrajt, G., Marcus, M.A., Leroux, H., Lemelle, L., Le, L., Lanzirotti, A., Langenhorst, F., Krot, A.N., Keller, L.P., Kearsley, A.T., Joswiak, D., Jacob, D., Ishii, H., Harvey, R., Hagiya, K., Grossman, L., Grossman, J.N., Graham, G.A., Gounelle, M., Gillet, P., Genge, M.J., Flynn, G., Ferroir, T., Fallon, S., Ebel, D.S., Dai, Z.R., Cordier, P., Clark, B., Chi, M., Butterworth, A.L., Brownlee, D.E., Bridges, J.C., Brennan, S., Brearley, A., Bradley, J.P., Bleuet, P., Bland, P.A., Bastien, R., 2006. Mineralogy and petrology of comet 81P/Wild 2 nucleus samples. Science 314, 1735-1739. 